ENSL-00315624

\title{
Lectures on Gauged Supergravity and Flux Compactifications
}

\author{
given at the RTN Winter School on \\ Strings, Supergravity and Gauge Theories, CERN, January 2008.
}

\author{
Henning Samtleben \\ Université de Lyon, Laboratoire de Physique, ENS Lyon, \\ 46 allée d'Italie, F-69364 Lyon CEDEX 07, France \\ henning.samtleben@ens-lyon.fr
}

\begin{abstract}
The low-energy effective theories describing string compactifications in the presence of fluxes are so-called gauged supergravities: deformations of the standard abelian supergravity theories. The deformation parameters can be identified with the various possible (geometric and non-geometric) flux components. In these lecture notes we review the construction of gauged supergravities in a manifestly duality covariant way and illustrate the construction in several examples.
\end{abstract}




\section{Contents}

1 Introduction $\quad 2$

2 Ungauged Supergravity — Symmetries and Dualities 5

2.1 Scalar sector . . . . . . . . . . . . . . . . 6 6 . . . . . . . . .

2.2 Vectors and antisymmetric $p$-forms . . . . . . . . . . . . . . . . 10

2.3 Self-duality in even dimensions . . . . . . . . . . . . . . . 13

3 Gauging Supergravity - Covariant Formulation 15

3.1 The embedding tensor . . . . . . . . . . . . . . . . 16

3.2 Deformed tensor gauge algebra . . . . . . . . . . . . . . [ . . . . . . . . . . .

3.3 The Lagrangian . . . . . . . . . . . . . . . . . . . . . . 27

4 Flux Compactifications - Examples 33

4.1 Higher-dimensional origin of symmetries . . . . . . . . . . . . 33

4.2 M-theory fluxes . . . . . . . . . . . . . . . . . . . . 37

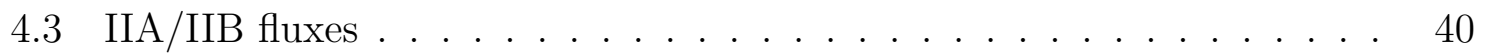

\section{Introduction}

Gauged supergravities have first been constructed in the early 1980's upon reconciling four-dimensional supergravity with maximal number of supercharges with the nonabelian gauge structure of Yang-Mills theories [1]. Soon after, the first construction was generalized to other (non-compact) gauge groups [2] and to higher dimensions [3], [4]. To date, gaugings are the only known supersymmetric deformations of maximal supergravity with the non-abelian gauge coupling constant acting as a deformation parameter. In recent years, these theories have reappeared in particular in the context of flux compactifications, see [5, 6] for reviews. Non-vanishing background fluxes for the higher-dimensional $p$-form tensor fields and so-called geometric fluxes twisting the internal geometry of the compactification manifold may likewise act as deformation parameters in the effective four-dimensional field theory. The resulting actions can be described in the framework of gauged supergravities with the resulting gauge groups typically being of the non-semisimple type.

In these lectures we will review the construction of gauged supergravities, i.e. we will address the problem of describing the general deformation of supergravity theories by coupling the abelian vector fields to charges assigned to the elementary fields. The general picture is sketched in figure 1, starting from eleven-dimensional supergravity [7] (or alternatively the ten-dimensional type IIB theory [8, 9]) the maximal supergravities 


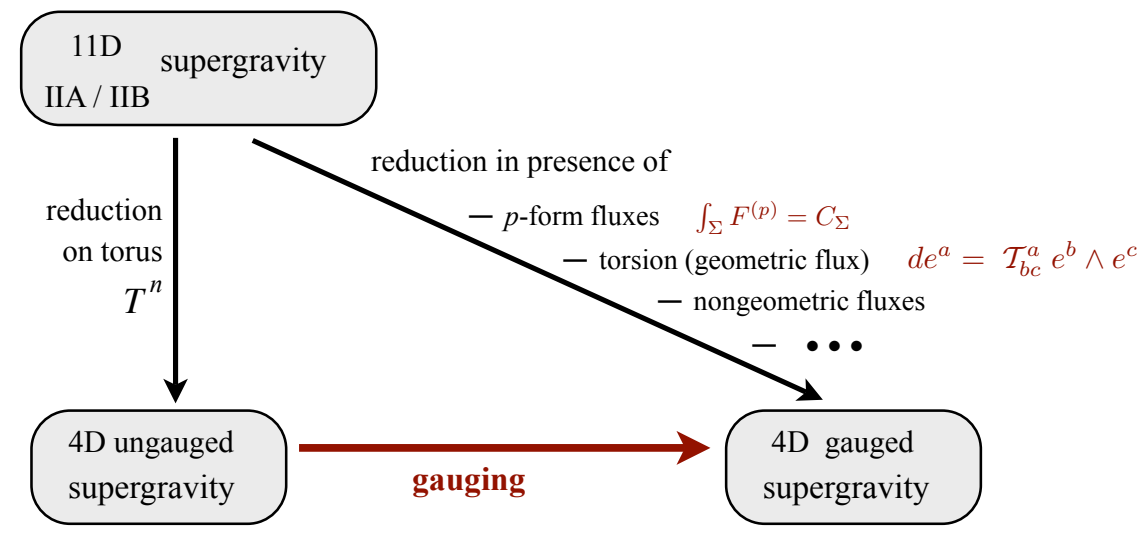

Figure 1: Gauged supergravities and flux compactifications.

in all lower dimensions are obtained by dimensional reduction on torus manifolds $T^{n}$ (the vertical arrow). Their characteristic properties include exceptionally large global symmetry groups and abelian gauge groups; e.g. for the maximal four-dimensional theory these are a global $\mathrm{E}_{7}$ and a local $\mathrm{U}(1)^{28}$ symmetry, respectively [10. None of the matter fields are charged under the abelian gauge group, hence the name of ungauged supergravity. Another distinct feature of these theories is their maximally supersymmetric Minkowski ground state in which all fields are massless.

Instead, one may consider more complicated compactifications (the diagonal arrow in figure 1), in which e.g. the torus is replaced by manifolds with more structure (such as spheres $S^{n}$ ), in which higher-dimensional $p$-form fields may acquire non-trivial background fluxes, in which the torus may be supplied with torsion, etc. All these compactifications lead to more complicated effective theories in four dimensions which typically come with non-abelian gauge symmetries under which the matter fields are charged, and which are referred to as gauged supergravities. In contrast to their ungauged counterparts, these theories typically come with a scalar potential which is a result of the more complicated internal geometry. This is one of the reasons that has triggered the interest in these compactifications: the scalar potential may support an effective cosmological constant, provide mass terms for the fields of the theory (moduli stabilization), describe scenarios of spontaneous supersymmetry breaking, etc., thereby accommodating many phenomenologically desirable properties. Except for very few examples, these gauged supergravities do no longer admit maximally supersymmetric groundstates in accordance with the fact that the presence of non-vanishing background fluxes typically breaks supersymmetry.

The most systematic approach to the construction and study of these gauged supergravities is by considering them as deformations of the ungauged theories obtained by simple torus reduction. This is depicted by the horizontal arrow in figure 1, with the flux and geometric parameters acting as deformation parameters. On the level 
of the four-dimensional theory, this construction selects a subgroup $\mathrm{G}_{0}$ of the global symmetry group $\mathrm{G}$ of the ungauged theory and promotes it to a local gauge symmetry by coupling it to the (formerly abelian) vector fields of the theory. As a result, the matter fields of the theory are charged under the new gauge symmetry. The first example of such a construction was the $\mathrm{SO}(8)$ gauged theory of [1] which describes the $S^{7}$ compactification of eleven-dimensional supergravity, with $\mathrm{SO}(8)$ properly embedded into the global $\mathrm{E}_{7}$ symmetry of the ungauged theory. In the context of flux compactifications, many other typically non-semisimple gaugings of this theory have been identified, some of which we will describe in the last section. Fortunately, all different gaugings can be described in a single covariant construction that is based on the underlying global symmetry group $\mathrm{G}$ of the ungauged theory. This framework, first developed in the context of three-dimensional supergravity [11, 12] and further shaped in [13, 14, 15], encodes the possible gaugings in an embedding tensor that describes the embedding of the gauge group into the global symmetry group, can be characterized group-theoretically, and turns out to entirely parametrize the action of the gauged supergravity. From the point of view of flux compactifications, this can be seen as a very compact way to group all the different possible flux (or deformation) parameters into a single tensorial object on which furthermore the action of the duality group is manifest. This will be the central theme of these lectures.

So far we have presented the picture for the supergravities with maximal number of supercharges and for definiteness we will throughout stick with the maximal or half-maximal theories whose structures are very rigid due to the large underlying global symmetries. We should stress however, that a large part of the structures and techniques to be presented directly apply to the supergravities with lower number of supercharges. E.g. in many applications the torus manifold in figure 1 would be replaced by a Calabi-Yau manifold such that the ungauged four-dimensional supergravity is no longer maximal but has only $\mathcal{N}=2$ supersymmetry. In complete analogy to the construction presented in the following, the effect of non-vanishing background fluxes can be accommodated by gauging certain global symmetries in these models leading to the same type of gauged supergravity in four dimensions. This has been confirmed in many explicit examples, see e.g. [16, 17, 18].

As a last point we mention that gauged supergravities have recently (re)appeared in other contexts as well which we will not further discuss in these lectures. Two important ones are the following:

- The supergravity regime of the bulk theory in the AdS/CFT correspondence [19] is generically described by a gauged supergravity. It is due to its scalar potential that the theory can support an AdS ground state. The corresponding gauge group is usually compact and corresponds to the R-symmetry group of the boundary theory. The prime-example is the five dimensional maximal $\mathrm{SO}(6)$ gauged supergravity of [3] which describes IIB supergravity compactified on $\operatorname{AdS}_{5} \times S^{5}$. Its scalar potential encodes non-trivial information about the four-dimensional 
SYM boundary theory, such as holographic RG flows and the anomalous conformal dimensions of operators [20, 21, 22].

- The structure of the gauged supergravities fits naturally with and gives further support to the proposals for the higher rank Kac-Moody symmetries $\mathrm{E}_{10}$ [23] and $\mathrm{E}_{11}$ 24], conjectured to underlie supergravity and string theory, As we shall discuss in section 3 , the field content of gauged supergravities is typically larger as compared to the ungauged theories, since the former naturally include a number of dual tensor fields and in particular the non-propagating antisymmetric $(D-1)$ and $D$-form tensor fields. This larger field content is precisely in accordance with certain decompositions of the representations of the extended infinite-dimensional Kac-Moody algebras [25, 26].

The structure of these lectures is straightforward: in section 2 we briefly review the structure of ungauged supergravities, in particular the role and the realization of their global symmetry groups G. In section 3 we describe the gauging of these theories in a formalism covariant under the symmetry group G. Finally, in section 4, we discuss the higher-dimensional origin of the gauged theories, in particular their application to the description of flux compactifications, and illustrate the connection with several examples.

\section{Ungauged Supergravity - Symmetries and Dualities}

In this section, we collect some of the pertinent facts about ungauged supergravity theories. The discussion will be rather brief and is not meant to be an exhaustive introduction to these theories - for which we refer to the many excellent reviews in the literature, see e.g. [27, 28, 29, 30, 31]. Rather, we will here focus onto those elements that prove to be important for the subsequent construction of gauged supergravities, notably the underlying structure of symmetries and dualities. We will mainly restrict to the bosonic sector of these theories, although its structure is of course to a large extent determined by the underlying supersymmetric extension.

The bosonic field content of standard supergravity theories consists of the metric $g_{\mu \nu}$, a set of scalar fields $\phi^{i}$, as well as vector fields $A_{\mu}^{M}$, and higher-rank antisym-

metric $p$-forms $B_{\nu_{1} \ldots \nu_{p}}^{I}$ of various ranks. Their dynamics is described in terms of a Lagrangian of the type

$$
e^{-1} \mathcal{L}_{\text {bos }}=-\frac{1}{2} R-\frac{1}{2} G_{i j}(\phi) \partial_{\mu} \phi^{i} \partial^{\mu} \phi^{j}-\frac{1}{4} \mathcal{M}_{M N}(\phi) F_{\mu \nu}^{M} F^{\mu \nu N}-\cdots,
$$

with $e=\sqrt{\left|\operatorname{det} g_{\mu \nu}\right|}$, and the abelian field strengths $F_{\mu \nu}^{\mathcal{M}} \equiv \partial_{\mu} A_{\nu}^{M}-\partial_{\nu} A_{\mu}^{M}$. The $\operatorname{dots}$ here refer to kinetic terms for the higher-rank $p$-forms and to possible topological terms. We will in these lectures always work in the Einstein frame, i.e. absorb possible dilaton prefactors of the Ricci scalar $R$ by conformal rescaling of the metric. 


\begin{tabular}{r||c|c|c|c|}
\multicolumn{1}{r|}{} & $\mathrm{G}_{\max }$ & $\mathrm{K}_{\max }$ & $\mathrm{G}_{\text {half }-\max }$ & $\mathrm{K}_{\text {half }-\max }$ \\
\hline 9 & $\mathrm{GL}(2)$ & $\mathrm{SO}(2)$ & $\mathrm{GL}(1) \times \mathrm{SO}(1,1+n)$ & $\mathrm{SO}(1+n)$ \\
8 & $\mathrm{SL}(2) \times \mathrm{SL}(3)$ & $\mathrm{SO}(2) \times \mathrm{SO}(3)$ & $\mathrm{GL}(1) \times \mathrm{SO}(2,2+n)$ & $\mathrm{SO}(2) \times \mathrm{SO}(2+n)$ \\
7 & $\mathrm{SL}(5)$ & $\mathrm{SO}(5)$ & $\mathrm{GL}(1) \times \mathrm{SO}(3,3+n)$ & $\mathrm{SO}(3) \times \mathrm{SO}(3+n)$ \\
6 & $\mathrm{SO}(5,5)$ & $\mathrm{SO}(5) \times \mathrm{SO}(5)$ & $\mathrm{GL}(1) \times \mathrm{SO}(4,4+n)$ & $\mathrm{SO}(4) \times \mathrm{SO}(4+n)$ \\
5 & $\mathrm{E}_{6(6)}$ & $\mathrm{USp}(8)$ & $\mathrm{GL}(1) \times \mathrm{SO}(5,5+n)$ & $\mathrm{SO}(5) \times \mathrm{SO}(5+n)$ \\
4 & $\mathrm{E}_{7(7)}$ & $\mathrm{SU}(8)$ & $\mathrm{SL}(2) \times \mathrm{SO}(6,6+n)$ & $\mathrm{SO}(2) \times \mathrm{SO}(6) \times \mathrm{SO}(6+n)$ \\
3 & $\mathrm{E}_{8(8)}$ & $\mathrm{SO}(16)$ & $\mathrm{SO}(8,8+n)$ & $\mathrm{SO}(8) \times \mathrm{SO}(8+n)$ \\
2 & $\mathrm{E}_{9(9)}$ & $\mathrm{K}\left(\mathrm{E}_{9}\right)$ & $\mathrm{SO}(8,8+n)^{(1)}$ & $\mathrm{K}\left(\mathrm{SO}(8,8+n)^{(1)}\right)$
\end{tabular}

Table 1: Global symmetry groups $\mathrm{G}$ and their compact subgroups $\mathrm{K}$ in maximal and halfmaximal supergravity in various dimensions. The subscripts in parentheses $\mathrm{E}_{N(N)}$ specify the particular real form of the exceptional groups; for maximal supergravity this is always the split form, i.e. the maximally non-compact form of the group. For $D=2$, the groups $\mathrm{E}_{9(9)}$ and $\mathrm{SO}(8,8+n)^{(1)}$ refer to the (centrally extended) affine extensions of the groups $\mathrm{E}_{8(8)}$ and $\mathrm{SO}(8,8+n)$, respectively, $\mathrm{K}(\mathrm{G})$ denotes their maximal compact subgroup.

The form of the Lagrangian (2.1) is essentially fixed by diffeomorphism and gauge covariance (upon restricting the dynamics to two-derivative terms). The "data" that remain to be specified are the scalar and the vector kinetic matrices $G_{i j}(\phi)$ and $\mathcal{M}_{M N}(\phi)$, respectively, as well as their counterparts for the higher-rank $p$-forms. In general, the form of these scalar-dependent matrices is highly constrained by supersymmetry. In the following we will mainly consider maximal and half-maximal supergravities (i.e. theories with 32 and 16 real supercharges, respectively) for which the possible couplings are extremely restrictive and organized by the structure of an underlying global symmetry group G. Most of the discussion straightforwardly extends to theories with a lower number of supercharges, in particular in those cases in which the underlying global symmetry group is still sufficiently large.

In the rest of this section, we will describe how the global symmetry group restricts the field content and determines the Lagrangian (2.1) without further explicit reference to supersymmetry. The $p$-forms and the scalar fields of the theory transform in linear and non-linear representations of $\mathrm{G}$, respectively, whereas the metric $g_{\mu \nu}$ is left invariant by the action of $\mathrm{G}$.

\subsection{Scalar sector}

The scalar fields $\phi^{i}$ in (half-)maximal supergravity are described by a $\mathrm{G} / \mathrm{K}$ coset space sigma-model, where $\mathrm{G}$ is the global symmetry group of the theory, collected in table 1 for various dimensions, and $\mathrm{K}$ is its maximal compact subgroup. A convenient formulation of this sigma-model has the scalar fields parametrize a G-valued matrix $\mathcal{V}$ (evaluated in some fundamental representation of $\mathrm{G}$ ) and makes use of the left-invariant 
current

$$
J_{\mu}=\mathcal{V}^{-1} \partial_{\mu} \mathcal{V} \in \mathfrak{g} \equiv \text { Lie G }
$$

In order to accommodate the coset space structure, $J_{\mu}$ is decomposed according to

$$
J_{\mu}=Q_{\mu}+P_{\mu}, \quad Q_{\mu} \in \mathfrak{k}, P_{\mu} \in \mathfrak{p}
$$

where $\mathfrak{k} \equiv$ Lie $\mathrm{K}$ and $\mathfrak{p}$ denotes its complement, i.e. $\mathfrak{g}=\mathfrak{k} \perp \mathfrak{p}$, orthogonal w.r.t. the Cartan-Killing form. The scalar Lagrangian is given by

$$
\mathcal{L}_{\text {scalar }}=-\frac{1}{2} e \operatorname{Tr}\left(P_{\mu} P^{\mu}\right) .
$$

It is invariant under global $\mathrm{G}$ and local $\mathrm{K}$ transformations acting as

$$
\delta \mathcal{V}=\Lambda \mathcal{V}-\mathcal{V} k(x), \quad \Lambda \in \mathfrak{g}, \quad k(x) \in \mathfrak{k}
$$

on the scalar matrix $\mathcal{V}$. Under these symmetries the currents $Q_{\mu}$ and $P_{\mu}$ transform according to

$$
\delta Q_{\mu}=-\partial_{\mu} k+\left[k, Q_{\mu}\right], \quad \delta P_{\mu}=\left[k, P_{\mu}\right]
$$

showing that the composite connection $Q_{\mu}$ behaves as a gauge field under K. As such it plays the role of a connection in the covariant derivatives of the fermion fields which transform linearly under the local K symmetry, e.g.

$$
D_{\mu} \psi_{\nu}^{i} \equiv \partial_{\mu} \psi_{\nu}^{i}-\frac{1}{4} \omega_{\mu}^{a b} \gamma_{a b} \psi_{\nu}^{i}-\left(Q_{\mu}\right)_{k}^{i} \psi_{\nu}^{k}
$$

for the gravitinos $\psi_{\nu}^{i}$, etc. Likewise, one defines $D_{\mu} \mathcal{V} \equiv \partial_{\mu} \mathcal{V}-Q_{\mu}=P_{\mu}$ for the scalar matrix $\mathcal{V}$. The current $P_{\mu}$ on the other hand transforms in a linear representation of $\mathrm{K}$, builds the K-invariant kinetic term (2.4) and may be used to construct K-invariant fermionic interaction terms in the Lagrangian.

The two symmetries 2.5 extend to the entire supergravity field content and play a crucial role in establishing the full supersymmetric action. They will furthermore be of vital importance in organizing the construction of the gauged theories described in the next section. The global $\mathfrak{g}$ transformations may be expanded as $\Lambda=\Lambda^{\alpha} t_{\alpha}$ into a basis of generators $t_{\alpha}$ satisfying standard Lie-algebra commutation relations

$$
\left[t_{\alpha}, t_{\beta}\right]=f_{\alpha \beta}^{\gamma} t_{\gamma}
$$

with structure constants $f_{\alpha \beta}$.

The local $\mathrm{K}$ symmetry is not a gauge symmetry associated with propagating gauge fields (the role of the gauge field is played by the composite connection $Q_{\mu}$ ), but simply takes care of the redundancy in parametrizing the coset space $\mathrm{G} / \mathrm{K}$. It is indispensable for the description of the fermionic sector, with the fermionic fields transforming in linear representations under $\mathrm{K}$. In particular, the scalar matrix $\mathcal{V}$ transforming 
as 2.5 can be employed to describe couplings between bosonic and fermionic fields, transforming under $\mathrm{G}$ and $\mathrm{K}$, respectively. To make this more explicit, it is useful to express 2.5 in indices as

$$
\delta \mathcal{V}_{M^{\underline{N}}}=\Lambda^{\alpha}\left(t_{\alpha}\right)_{M}^{K} \mathcal{V}_{K}^{\underline{N}}-\mathcal{V}_{M^{\underline{K}}} k_{\underline{K}}^{\underline{N}}
$$

with G-generators $\left(t_{\alpha}\right)_{M}{ }^{K}$ and the underlined indices $\underline{K}, \underline{N}$ referring to their transformation behavior under the subgroup K. The matrix $\mathcal{V}_{M} \underline{N}$ allows to construct couplings of e.g. a bosonic field strength $F_{\mu \nu}^{M}$ transforming in the associated fundamental representation of $\mathrm{G}$ to the fermionic fields according to (schematically)

$$
F^{M} \mathcal{V}_{M^{\underline{N}}}(\bar{\psi} \psi)_{\underline{N}}, \quad \text { etc. }
$$

where $(\bar{\psi} \psi)_{\underline{N}}$ denotes the projection of the fermionic bilinear onto some K-subrepresentation in the corresponding tensor product of K-representations.

It is often convenient to fix the local $\mathrm{K}$ symmetry by adopting a particular form of the matrix $\mathcal{V}$, i.e. choosing a particular set of coset representatives. In this case, any global G-transformation in (2.5) needs to be accompanied by a compensating Ktransformation

$$
\delta \mathcal{V}=\Lambda \mathcal{V}-\mathcal{V} k_{\Lambda}
$$

where $k_{\Lambda}$ depends on $\Lambda$ (and on $\mathcal{V}$ ) in order to restore the particular gauge choice, i.e. to preserve the chosen set of coset representatives. This defines a non-linear representation of $\mathrm{G}$ on the $(\operatorname{dim} \mathrm{G}-\operatorname{dim} \mathrm{K})$ coordinates of the coset space, i.e. on the physical scalar fields. Likewise, it provides a non-linear realization of the group $G$ on the fermion fields via the compensating transformation $k_{\Lambda}$. Two prominent gauge fixings are the following:

- unitary gauge: in which the matrix $\mathcal{V}$ is taken of the form

$$
\mathcal{V}=\exp \left\{\phi^{a} Y_{a}\right\}
$$

where the non-compact generators $Y_{a}$ span the space $\mathfrak{p}$. In this gauge, the $\phi^{a}$ transform in a linear representation of $\mathrm{K} \subset \mathrm{G}$, thus global K-invariance of the Lagrangian remains manifest. The current $P_{\mu}=P_{\mu}^{a} Y^{a}$ takes the form $P_{\mu}^{a}=$ $\partial_{\mu} \phi^{a}+\ldots$, where dots refer to higher order contributions. This shows that the kinetic term (2.4) is manifestly ghost-free with $G_{a b}(\phi) \propto \delta_{a b}+\ldots$. It is here that the importance of $\mathrm{K}$ being the maximal compact subgroup of $\mathrm{G}$ shows up.

- triangular gauge: in which the matrix $\mathcal{V}$ is taken of the form

$$
\mathcal{V}=\exp \left\{\phi^{m} N_{m}\right\} \exp \left\{\phi^{\lambda} h_{\lambda}\right\}
$$

where $\lambda=1, \ldots$, rank $\mathrm{G}$, labels a set of Cartan generators $h_{\lambda}$ of $\mathfrak{g}$ and the $N_{m}$ form a set of nilpotent generators such that the algebra spanned by $\left\{h_{\lambda}, N_{m}\right\}$ 
constitutes a Borel subalgebra of $\mathfrak{g}$. With suitable choice of the Borel subalgebra, it is in this gauge that a possible higher-dimensional origin of the theories becomes the most transparent. The grading associated with the chosen Borel subalgebra is related to the charges of the fields under rescaling of the volume of the internal compactification manifold. We shall illustrate this in section 4 in several examples, see [32] for a systematic discussion for the maximal theories and their eleven-dimensional origin.

E.g. the scalar sector of the maximal $(\mathcal{N}=8)$ supergravity in $D=4$ space-time dimensions is described by the coset space $\mathrm{E}_{7(7)} / \mathrm{SU}(8)$. The eleven-dimensional origin of the fields can be identified in the triangular gauge associated with the GL(7) grading of $\mathrm{E}_{7(7)}$. A type IIB origin of the fields on the other hand is identified in the triangular gauge associated with a particular $\mathrm{GL}(6) \times \mathrm{SL}(2)$ grading. We shall come back to this in section 4. For the half-maximal $(\mathcal{N}=4)$ supergravity in $D=4$ dimensions, the scalar sector is described by the coset space

$$
\mathrm{G} / \mathrm{K}=\mathrm{SL}(2) / \mathrm{SO}(2) \times \mathrm{SO}(6,6+n) /(\mathrm{SO}(6) \times \mathrm{SO}(6+n)),
$$

where $n$ refers to the number of vector multiplets in the ten-dimensional type-I theory, from which this theory is obtained by torus reduction. The ten-dimensional origin of the fields is identified in the triangular gauge associated with the GL(6) grading of $\mathrm{SO}(6,6)$.

In order to construct the full supersymmetric action of the theory, it is most convenient to keep the local $\mathrm{K}$ gauge freedom. When discussing only the bosonic sector of the theory, it is often functional to formulate the theory in terms of manifestly Kinvariant objects. E.g. the scalar fields can equivalently be described in terms of the positive definite symmetric scalar matrix $\mathcal{M}$ defined by

$$
\mathcal{M} \equiv \mathcal{V} \Delta \mathcal{V}^{\mathrm{T}}
$$

where $\Delta$ is a constant K-invariant positive definite matrix (e.g. for the coset space $\mathrm{SL}(N) / \mathrm{SO}(N)$, with $\mathcal{V}$ in the fundamental representation, $\Delta$ is simply the identity matrix). The matrix $\mathcal{M}$ is manifestly $\mathrm{K}$-invariant and transforms under $\mathrm{G}$ as

$$
\delta \mathcal{M}=\Lambda \mathcal{M}+\mathcal{M} \Lambda^{\mathrm{T}},
$$

while the Lagrangian 2.4 takes the form

$$
\mathcal{L}_{\text {scalar }}=\frac{1}{8} \operatorname{Tr}\left(\partial_{\mu} \mathcal{M} \partial^{\mu} \mathcal{M}^{-1}\right) .
$$

To finish this section, let us evaluate the general formulas for the simplest non-trivial coset-space $\mathrm{SL}(2) / \mathrm{SO}(2)$ which appears in the matter sector of several supergravity theories. With the $\mathfrak{s l}(2)$ generators given by

$$
\mathbf{h}=\left(\begin{array}{cc}
1 & 0 \\
0 & -1
\end{array}\right), \quad \mathbf{e}=\left(\begin{array}{cc}
0 & 1 \\
0 & 0
\end{array}\right), \quad \mathbf{f}=\left(\begin{array}{cc}
0 & 0 \\
1 & 0
\end{array}\right)
$$


the matrix $\mathcal{V}$ in triangular gauge $(2.13)$ is given as

$$
\mathcal{V}=\mathrm{e}^{C \mathbf{e}} \mathrm{e}^{\phi \mathbf{h}}=\left(\begin{array}{cc}
1 & C \\
0 & 1
\end{array}\right)\left(\begin{array}{cc}
\mathrm{e}^{\phi} & 0 \\
0 & \mathrm{e}^{-\phi}
\end{array}\right)
$$

Evaluating the non-linear realization (2.11) of SL(2) on these coset coordinates $C, \phi$ leads to

$$
\delta_{\mathbf{h}} \phi=1, \quad \delta_{\mathbf{h}} C=2 C, \quad \delta_{\mathbf{e}} C=1, \quad \delta_{\mathbf{f}} \phi=-C, \quad \delta_{\mathbf{f}} C=\mathrm{e}^{4 \phi}-C^{2} .
$$

This shows that $\mathbf{h}$ acts as a scaling symmetry on the fields, whereas e acts as a shift symmetry on $C$, and $\mathbf{f}$ is realized non-linearly. This toy example exhibits already all the generic features of the global G-symmetries that we will meet in more generality in section 4.1 below.

The matrix $\mathcal{M}$ for this model can be computed from 2.15 with $\Delta=\mathbb{I}_{2}$, and is most compactly expressed in terms of a complex scalar field $\tau=C+i \mathrm{e}^{2 \phi}$, giving rise to

$$
\mathcal{M}=\frac{1}{\Im \tau}\left(\begin{array}{cc}
|\tau|^{2} & \Re \tau \\
\Re \tau & 1
\end{array}\right)
$$

while the kinetic term (2.4) takes the form

$$
e^{-1} \mathcal{L}_{\text {scalar }}=-\partial_{\mu} \phi \partial^{\mu} \phi-\frac{1}{4} e^{-4 \phi} \partial_{\mu} C \partial^{\mu} C=-\frac{1}{4(\Im \tau)^{2}} \partial_{\mu} \tau \partial^{\mu} \tau^{*}
$$

It is manifestly invariant under the scaling and shift symmetries of 2.20), whereas invariance under the non-linear action of $\mathbf{f}$ is not obvious and sometimes referred to as a hidden symmetry. In terms of $\tau$, the action of a finite $\operatorname{SL}(2)$ group transformation can be given in the compact form

$$
\tau \rightarrow \frac{a \tau+b}{c \tau+d}, \quad \text { for } \quad \exp (\Lambda)=\left(\begin{array}{ll}
a & b \\
c & d
\end{array}\right) \in \mathrm{SL}(2)
$$

\subsection{Vectors and antisymmetric $p$-forms}

The $p$-forms in ungauged supergravity transform in (typically irreducible) linear representations of the global symmetry group G. E.g. while the scalar fields transform under $\mathrm{G}$ as 2.5 , the transformation of the vector fields $A_{\mu}^{M}\left(M=1, \ldots, n_{\mathrm{v}}\right)$ is given by

$$
\delta A_{\mu}^{M}=-\Lambda^{\alpha}\left(t_{\alpha}\right)_{N}{ }^{M} A_{\mu}^{N}
$$

where $\left(t_{\alpha}\right)_{N}{ }^{M}$ denote the generators of $\mathfrak{g}$ in a fundamental representation $\mathcal{R}_{\mathrm{v}}$ with $\operatorname{dim} \mathcal{R}_{\mathrm{v}}=n_{\mathrm{v}}$. Similarly, the higher-rank $p$-forms transform in particular representations of $\mathrm{G}$. The $p$-form field content of the ungauged maximal supergravities in various dimensions is determined by supersymmetry and collected in table 2 . 


\begin{tabular}{c|c||c|c|c|c|}
$D$ & $\mathrm{G}$ & 0 & 1 & 2 & 3 \\
\hline 9 & $\mathrm{GL}(2)$ & $\mathbf{1}^{0}+\mathbf{3}^{0}-1$ & $\mathbf{1}^{-4}+\mathbf{2}^{+3}$ & $\mathbf{2}^{-1}$ & $\mathbf{1}^{+2}$ \\
8 & $\mathrm{SL}(2) \times \mathrm{SL}(3)$ & $(\mathbf{3}-1, \mathbf{1})+(\mathbf{1}, \mathbf{8}-3)$ & $\left(\mathbf{2}, \mathbf{3}^{\prime}\right)$ & $(\mathbf{1}, \mathbf{3})$ & $(\mathbf{2}, \mathbf{1})$ \\
7 & $\mathrm{SL}(5)$ & $\mathbf{2 5}-15$ & $\mathbf{1 0}^{\prime}$ & $\mathbf{5}$ & \\
6 & $\mathrm{SO}(5,5)$ & $\mathbf{4 5}-30$ & $\mathbf{1 6}_{c}$ & $\mathbf{1 0}$ & \\
5 & $\mathrm{E}_{6(6)}$ & $\mathbf{7 8}-36$ & $\mathbf{2 7 ^ { \prime }}$ & & \\
4 & $\mathrm{E}_{7(7)}$ & $\mathbf{1 3 3}-63$ & $\mathbf{5 6}$ & & \\
3 & $\mathrm{E}_{8(8)}$ & $\mathbf{2 4 8}-120$ & & &
\end{tabular}

Table 2: The $p$-form field content in ungauged maximal supergravity organizes into Grepresentations. The physical scalars $(p=0)$ descend from the adjoint representation of $\mathrm{G}$ upon eliminating ( $\operatorname{dim} \mathrm{K}$ ) of them by fixing the local $\mathrm{K}$ freedom, cf. (2.11). The framed representations appearing in the even dimensions refer to the peculiarity concerning the $(D / 2-1)$ forms, of which only half appear in the Lagrangian and carry propagating degrees of freedom, as discussed in subsection 2.3 below.

An invariant action for the vector fields is given by

$$
\mathcal{L}_{\text {kin }}=-\frac{1}{4} e \mathcal{M}_{M N} F_{\mu \nu}^{M} F^{\mu \nu N}
$$

with the abelian field strength $F_{\mu \nu}^{\mathcal{M}} \equiv \partial_{\mu} A_{\nu}^{M}-\partial_{\nu} A_{\mu}^{M}$ and the scalar dependent positive definite matrix $\mathcal{M}_{M N}$ defined in 2.15). This action is manifestly invariant under $\mathrm{G}$ with $\mathcal{M}$ transforming as 2.16). To be precise, the action 2.25 is only relevant for the vector fields in $D>4$ dimensions, while in $D=4$ space-time dimensions the story is somewhat more complicated as a consequence of electric/magnetic duality as we shall briefly review in the next subsection. Similarly, the kinetic terms for higherrank $p$-forms $B_{\nu_{1} \ldots \nu_{p}}^{I}$ (with $p<[(D-1) / 2]$ ) are governed by the positive definite scalar matrices (2.15) evaluated in the corresponding representations

$$
\mathcal{L}_{\text {kin }}=-\frac{1}{2(p+1) !} \mathcal{M}_{I J} F_{\nu_{1} \ldots \nu_{p+1}}^{I} F^{\nu_{1} \ldots \nu_{p+1} J}, \quad \text { etc. }
$$

with the abelian field strength ${ }^{1} F_{\nu_{1} \ldots \nu_{p+1}}^{I}=(p+1) \partial_{\left[\nu_{1}\right.} B_{\left.\nu_{2} \ldots \nu_{p} \nu_{p+1}\right]}^{I}$.

An important ingredient in the construction of supergravity theories by dimensional reduction which will also be of relevance in the construction of the gaugings below is the on-shell duality between massless $p$-forms and $(D-p-2)$-forms in $D$ space-time dimensions. This simply reflects the fact that these forms carry the same representation under the little group $\mathrm{SO}(D-2)$. Specifically, it follows from 2.26 that the field equation of a $p$-form $B^{I}$ to lowest order in the fields take the form

$$
\partial^{\mu}\left(\mathcal{M}_{I J} F_{\mu \nu_{1} \ldots \nu_{p}}^{J}\right)=0
$$

The full field equations receive higher-order terms in the fermions as well as contributions from possible topological terms. At the same time, the abelian field strength $F^{I}$

\footnotetext{
${ }^{1}$ Throughout, when antisymmetrizing indices $\left[\mu_{1} \ldots \mu_{p}\right]$, we use the normalization with total weight one, i.e. $X_{[\mu \nu]}=\frac{1}{2}\left(X_{\mu \nu}-X_{\nu \mu}\right)$, etc.
} 
is subject to the Bianchi identity

$$
\partial_{\left[\nu_{1}\right.} F_{\left.\nu_{2} \ldots \nu_{p+2}\right]}^{I}=0 .
$$

In terms of the dual field strength

$$
G_{\mu_{1} \ldots \mu_{D-p-1} I} \equiv \frac{e}{(p+1) !} \varepsilon_{\mu_{1} \ldots \mu_{D-p-1} \nu_{1} \ldots \nu_{p+1}} \mathcal{M}_{I J} F^{\nu_{1} \ldots \nu_{p+1} J},
$$

the equations (2.27) and (2.28) take the form

$$
\partial_{\left[\mu_{1}\right.} G_{\left.\mu_{2} \ldots \mu_{D-p}\right] I}=0, \quad \text { and } \partial^{\mu}\left(\mathcal{M}^{I J} G_{\mu \nu_{1} \ldots \nu_{D-p-2} J}\right)=0,
$$

respectively, i.e. equations of motion and Bianchi identities exchange their roles and locally we can define the dual $(D-p-2)$-forms $C_{I}$ by

$$
G_{\mu_{1} \ldots \mu_{D-p-1} I} \equiv(D-p-1) \partial_{\left[\mu_{1}\right.} C_{\left.\mu_{2} \ldots \mu_{D-p-1}\right] I} .
$$

Dynamics of the massless $p$-forms $B^{I}$ can thus equivalently be described in terms of their dual ( $D-p-2)$-forms $C_{I}$, transforming in the dual representation under the global symmetry G. This equivalence extends to the full non-linear theory, i.e. in presence of Chern-Simons terms and couplings to the fermion fields.

As a result, there are in general several different off-shell formulations of a given ungauged supergravity which are on-shell equivalent only after dualizing part of their field content according to (2.29). It may not always be possible to get rid of all $p$-forms by this dualization, as the presence of topological terms with explicit appearance of the gauge fields can prevent the elimination of these fields by virtue of (2.29). There is however always a version of the theory in which all forms are dualized to lowest possible degree. This is the version in which the largest global symmetry group $\mathrm{G}$ is manifest, and the $p$-forms couple with kinetic terms (2.25) and (2.26), respectively. We will see in section 3 that all gaugings of supergravity can be obtained as deformations of this particular version of the ungauged theory.

Let us finally mention that the duality $(2.29)$ naturally extends to the scalar fields $(p=0)$, which are hence on-shell dual to $(D-2)$-forms. Due to the non-linear coupling of scalar fields discussed in section 2.1, the representation assignment is slightly different: $(D-2)$-forms generically transform in the full adjoint representation of the global symmetry group $\mathrm{G}$ with 2.29 replaced by

$$
G_{\mu_{1} \ldots \mu_{D-1} \alpha} \equiv e \varepsilon_{\mu_{1} \ldots \mu_{D-1} \nu} j_{\alpha}^{\nu},
$$

where $j_{\alpha}^{\nu}$ is the conserved Noether-current associated with the symmetry generated by $t_{\alpha}$. Again, this duality cannot be used to eliminate all scalar fields (as e.g. the scalar dependence of (2.4) cannot be expressed exclusively in terms of the $j_{\alpha}^{\nu}$ ), but only those fields on which the action of $\mathrm{G}$ is realized as a shift isometry $\phi^{i} \rightarrow \phi^{i}+c^{i}$. The apparent mismatch between the $(\operatorname{dim} G-\operatorname{dim} K)$ physical scalar fields and the number of $(D-2)$-forms defined in (2.32) is explained by the fact that not all the Noether currents $j_{\alpha}$ are independent: it follows from the structure of the coset space sigma-model that $\mathcal{V}^{-1}\left(j_{\alpha} t^{\alpha}\right) \mathcal{V} \in \mathfrak{p}$ for the Noether current associated with 2.4. This implies $(\operatorname{dim} \mathrm{K})$ linear constraints on the fields strengths $G_{\alpha}$. 


\subsection{Self-duality in even dimensions}

Employing the on-shell duality (2.29) one can always achieve a formulation of the theory in which all forms are dualized to a degree $p \leq[(D-1) / 2]$ and appear with the kinetic terms (2.25), 2.26). A subtlety arises in even dimensions $D=2 K$ for the coupling of the $(K-1)$-forms. Due to the duality $(2.29)$ between $(K-1)$-forms and $(K-1)$-forms, these forms appear in pairs $\left(B^{\Lambda}, B_{\Lambda}\right)$ of which only the first half enters the Lagrangian and carries propagating degrees of freedom while the other half is defined as their on-shell duals. For the maximal theories, one observes that only together the forms $B^{\Lambda}$ and their on-shell duals $B_{\Lambda}$ transform in a $2 m$-dimensional irreducible linear representation $B^{P}=\left(B^{\Lambda}, B_{\Lambda}\right)$ of the symmetry group $\mathrm{G}$, shown in table 2 . As a consequence, in even dimensions $\mathrm{G}$ is only realized as an on-shell symmetry. E.g. the $\mathcal{N}=8$ supergravity multiplet in $D=4$ dimensions carries 28 vector fields which show up in the Lagrangian, but it is only together with their 28 magnetic duals that they form the fundamental $\mathbf{5 6}$ representation of $\mathrm{G}=\mathrm{E}_{7(7)}[10$.

The analogue of the duality equation $(2.29)$ in this case is the on-shell G-covariant twisted self-duality equation 32 .

$$
F_{\nu_{1} \ldots \nu_{K}}^{P}=-\frac{e}{K !} \varepsilon_{\nu_{1} \ldots \nu_{K} \mu_{1} \ldots \mu_{K}} \Omega^{P Q} \mathcal{M}_{Q R} F^{\mu_{1} \ldots \mu_{K} R},
$$

for the G-covariant abelian field strength $F^{P}$, the symmetric matrix $\mathcal{M}_{P Q}$ from 2.15) evaluated in the corresponding $2 m$-dimensional representation of $\mathrm{G}$, and the matrix $\Omega^{P Q}$ given by

$$
\Omega^{P Q} \equiv\left(\begin{array}{cc}
0 & \mathbb{I}_{m} \\
\epsilon \mathbb{I}_{m} & 0
\end{array}\right), \quad \text { with } \epsilon=(-1)^{K+1}
$$

Consistency of 2.33 requires that the matrix $\Omega^{P Q} \mathcal{M}_{Q R}$ squares to $\epsilon$, such that the total operator acting on $F^{P}$ on the r.h.s. of this equation squares to the identity. This translates into the condition

$$
\mathcal{M}_{I K} \Omega^{K L} \mathcal{M}_{L J}=\Omega_{I J},
$$

i.e. requires the matrix $\mathcal{M}_{P Q}$ to be symplectic/orthogonal for $K$ even/odd, respectively. Indeed, table 1 shows that the global symmetry groups $\mathrm{G}$ in even dimensions can be embedded into $\operatorname{Sp}(m, m)$ and $\mathrm{SO}(m, m)$, for $K$ even/odd, respectively. For the nonsimple groups appearing in the list it is sufficient that the factor under which the $(K-1)$-forms transform non-trivially can be embedded into $\mathrm{Sp}(m, m)$ or $\mathrm{SO}(m, m)$, respectively. E.g. for the maximal theory in $D=8$, it is the $\mathrm{SL}(2) \sim \operatorname{Sp}(1,1)$ factor which mixes three-forms with their on-shell duals [33]. In $D=6$ dimensions we identify $\mathrm{SO}(5,5)$, and $\mathrm{GL}(1) \sim \mathrm{SO}(1,1)$, respectively, while in $D=4$ dimensions, (2.35) is ensured by the symplectic embeddings $\mathrm{E}_{7(7)} \subset \mathrm{Sp}(28,28)$, and $\mathrm{SL}(2) \times \mathrm{SO}(6,6+n) \subset$ $\operatorname{Sp}(12+n, 12+n)$, respectively. As $\Omega$ is a group-invariant tensor, equation 2.33 is manifestly G-covariant. Let us mention that for odd $K$ there are theories in which 
the eigenvalues of $\Omega^{P Q} \mathcal{M}_{Q R}$ do not come in real pairs \pm 1 , such that $\Omega$ does not take the form (2.34). These theories (which include the ten-dimensional IIB theory and the six-dimensional chiral theories with tensor multiplets) do not admit an action and we shall not consider them in the rest of this section.

In order to lift equation 2.33 to an action, one employs the split $B^{P}=\left(B^{\Lambda}, B_{\Lambda}\right)$ and constructs the action in terms of half of the fields $B^{\Lambda}$ considered as independent propagating (electric) fields, while the $B_{\Lambda}$ are defined via 2.33) as their on-shell (magnetic) duals [34]. To lowest order in the fields, the proper Lagrangian is given by

$$
\mathcal{L}_{\text {kin }}=\frac{1}{2 K !} e \mathcal{I}_{\Lambda \Sigma}(\phi) F_{\nu_{1} \ldots \nu_{K}}^{\Lambda} F^{\nu_{1} \ldots \nu_{K} \Sigma}+\frac{1}{2(K !)^{2}} \varepsilon^{\mu_{1} \ldots \mu_{K} \nu_{1} \ldots \nu_{K}} \mathcal{R}_{\Lambda \Sigma}(\phi) F_{\mu_{1} \ldots \mu_{K}}^{\Lambda} F_{\nu_{1} \ldots \nu_{K}}^{\Sigma},
$$

in terms of the $m$ abelian field strengths $F^{\Lambda}$, with the kinetic matrices $\mathcal{I}_{\Lambda \Sigma}(\phi)$ and $\mathcal{R}_{\Lambda \Sigma}(\phi)$ related to the matrix $\mathcal{M}_{P Q}$ as

$$
\mathcal{M}_{P Q} \equiv-\left(\begin{array}{cc}
\mathcal{I}-\epsilon \mathcal{R I}^{-1} \mathcal{R} & \epsilon \mathcal{R I}^{-1} \\
-\mathcal{I}^{-1} \mathcal{R} & \mathcal{I}^{-1}
\end{array}\right)
$$

Indeed, it is easy to verify that an arbitrary symmetric matrix $\mathcal{M}$ satisfying (2.35) can be parametrized as 2.37) in terms of two matrices $\mathcal{I}_{\Lambda \Sigma}=\mathcal{I}_{\Sigma \Lambda}$ and $\mathcal{R}_{\Lambda \Sigma}=-\epsilon \mathcal{R}_{\Sigma \Lambda}$, obeying the correct symmetry properties according to their appearance in (2.36). Moreover, $\mathcal{I}_{\Lambda \Sigma}$ is negative definite, such that the kinetic term in 2.36 comes with the correct sign.

The field equations implied by the Lagrangian (2.36) are conveniently expressed in terms of the dual field strength defined as

$$
G_{\mu_{1} \ldots \mu_{K} \Lambda} \equiv(-1)^{K+1} \varepsilon_{\mu_{1} \ldots \mu_{K} \nu_{1} \ldots \nu_{K}} \frac{\delta \mathcal{L}}{\delta F_{\nu_{1} \ldots \nu_{K}}^{\Lambda}} .
$$

as

$$
\partial_{\left[\mu_{1}\right.} G_{\left.\mu_{2} \ldots \mu_{K+1}\right] \Lambda}=0,
$$

allowing for the introduction of the $m$ dual $(K-1)$-forms $B_{\Lambda}$ according to (2.31), in terms of which Bianchi identities and field equations exchange their roles as in the previous section. Upon manipulation of $(2.38)$ one recovers the manifestly G-covariant form of the field equations 2.33 with $F^{P}=\left(F^{\Lambda}, G_{\Lambda}\right)$. The action 2.36 can be extended to the full non-linear theory, including higher order topological terms and fermionic fields.

Equation (2.37) shows that the linear action of $\mathrm{G}$ on $\mathcal{M} 2.16)$ generically translates into a non-linear action on the kinetic matrices $\mathcal{I}, \mathcal{R}$. Moreover, the action of $\mathrm{G}$ mixes the components of the vector $\left(B^{\Lambda}, B_{\Lambda}\right)$ and thus the forms appearing in 2.36) with their on-shell duals. As a consequence, $G$ is not a symmetry of the Lagrangian and only realized on-shell, as is manifest in 2.33). Only its subgroup corresponding to triangular generators

$$
\left(t_{\alpha}\right)_{P}{ }^{Q}=\left(\begin{array}{cc}
* & * \\
0 & *
\end{array}\right)
$$


is realized as an off-shell symmetry of the Lagrangian.

Different electric/magnetic splits $B^{K} \rightarrow\left(B^{\Lambda}, B_{\Lambda}\right)$ correspond to different electric frames and are related by symplectic/orthogonal rotation. These give rise to different off-shell formulations which are on-shell equivalent. In particular, the off-shell symmetry group depends on the particular choice of the electric frame.

An example of these structures is the half-maximal theory in $D=4$ dimensions with coset space (2.14) whose vector fields $A_{\mu}^{m \alpha}$ transform in the bifundamental representation of $\mathrm{SO}(6,6+n) \times \mathrm{SL}(2)$. A convenient electric/magnetic split is $A^{m \alpha} \rightarrow\left(A^{m+}, A^{m-}\right)$ breaking up the $\mathrm{SL}(2)$ doublet index $\alpha$. With the matrix $\mathcal{M}_{m \alpha, n \beta}$ of $(2.15)$ factorizing according to

$$
\mathcal{M}_{m \alpha, n \beta}=\mathcal{M}_{m n} \mathcal{M}_{\alpha \beta}
$$

into an $\mathrm{SO}(6,6+n)$ matrix $\mathcal{M}_{m n}$ and the $\mathrm{SL}(2)$ matrix $\mathcal{M}_{\alpha \beta}$ of (2.21), the Lagrangian (2.36) is found via (2.37) to be

$$
\mathcal{L}_{\text {kin }}=-\frac{1}{4}\left(e \Im \tau \mathcal{M}_{m n} F_{\mu \nu}^{m+} F^{\mu \nu n+}+\frac{1}{2} \varepsilon^{\mu \nu \sigma \tau} \Re \tau \eta_{m n} F_{\mu \nu}^{m+} F_{\sigma \tau}^{n+}\right),
$$

with the $\mathrm{SO}(6,6+n)$ invariant metric $\eta_{m n}$. This is an $\mathrm{SO}(6,6+n)$ covariant electric frame in which the SL(2) global symmetry is realized only on-shell. In other frames, the full SL(2) may be elevated to an off-shell symmetry, but only a GL(6) subgroup of $\mathrm{SO}(6,6+n)$ remains realized off-shell.

Let us finally mention that the case of $D=2$ supergravity is particularly subtle, as the self-duality of forms discussed in this section applies to the scalar fields of the theory. The formalism thus needs to be merged with the non-linear realization of the scalar isometries discussed in section 2.1. As a result, the duality between scalar fields is not of the simple type as for the $p$-forms, but rather leads to an infinite chain of mutually dual scalar fields, on which the infinite-dimensional global symmetry group $G$ can be linearly realized. See [35, 36, 37, 38, 39] for details.

\section{Gauging Supergravity - Covariant Formulation}

In the last section, we have reviewed how the field content and the action of ungauged supergravity are organized by the global symmetry group G. Scalar fields and $p$-form fields transform in a non-linear and in linear representations of $G$, respectively. We will now discuss the gaugings of the theory. I.e. according to the general discussion of section 1 we will select a subgroup $\mathrm{G}_{0} \subset \mathrm{G}$ and promote it to a local symmetry. This can be considered as a deformation of the ungauged theory and we shall discuss which additional couplings have to be imposed along the way. We will employ the covariant formalism of [11, 12, 13, 14, 15] in which the gaugings are encoded in the embedding tensor which may be characterized group-theoretically. 


\subsection{The embedding tensor}

As we have reviewed in the last section, under the non-abelian global symmetry group $\mathrm{G}$, the bosonic fields of ungauged supergravity transform as

$$
\begin{aligned}
& \qquad \mathcal{V}=\Lambda^{\alpha} t_{\alpha} \mathcal{V}, \\
& \delta A_{\mu}^{M}=-\Lambda^{\alpha}\left(t_{\alpha}\right)_{N}{ }^{M} A_{\mu}^{N}, \\
& \text { etc. }
\end{aligned}
$$

with constant parameters $\Lambda^{\alpha}, \alpha=1, \ldots, \operatorname{dim} \mathrm{G}$. In addition, the $n_{\mathrm{v}}$ vector fields in the theory possess the standard abelian gauge symmetry $\mathrm{U}(1)^{n_{\mathrm{v}}}$ :

$$
\delta A_{\mu}^{M}=\partial_{\mu} \Lambda^{M}
$$

with coordinate-dependent parameters $\Lambda^{M}=\Lambda^{M}(x)$. Similarly, higher-rank $p$-forms appear with the corresponding abelian tensor gauge symmetry.

Gauging corresponds to promoting a subgroup $\mathrm{G}_{0} \subset \mathrm{G}$ to a local symmetry. This subgroup can be defined by selecting a subset of generators within the global symmetry algebra $\mathfrak{g}=$ Lie $\mathrm{G}$. Denoting these generators by $X_{M}$, the associated symmetries can be made local by introducing standard covariant derivatives according to

$$
\partial_{\mu} \longrightarrow D_{\mu} \equiv \partial_{\mu}-g A_{\mu}^{M} X_{M}
$$

where we also introduce the gauge coupling constant $g$. A general set of $n_{\mathrm{v}}$ generators in $\mathfrak{g}$ can be described as

$$
X_{M} \equiv \Theta_{M}^{\alpha} t_{\alpha} \in \mathfrak{g}
$$

by means of a constant tensor $\Theta_{M}{ }^{\alpha}$, the embedding tensor, which describes the explicit embedding of the gauge group $\mathrm{G}_{0}$ into the global symmetry group $\mathrm{G}$. For the moment, we can simply consider this object as a constant $\left(n_{\mathrm{v}} \times \operatorname{dim} \mathrm{G}\right)$ matrix with its two indices $M$ and $\alpha$ in a fundamental and the adjoint representation of $\mathrm{G}$, respectively. It combines the full set of deformation parameters. The dimension of the gauge group is given by the rank of the matrix $\Theta_{M}{ }^{\alpha}$.

The advantage of explicitly parametrizing the gauge group generators as in (3.4) is, that this allows to keep the entire construction formally G-covariant. As it will turn out, the gauging can be entirely parametrized in terms of the embedding tensor $\Theta_{M}{ }^{\alpha}$. The deformed equations of motion remain manifestly G-covariant if the embedding tensor is treated as a spurionic object that simultaneously transforms under $\mathrm{G}$ according to the structure of its indices. It is only upon specifying a particular choice for $\Theta_{M}{ }^{\alpha}$ that we select a particular gauge group $\mathrm{G}_{0}$, and the global symmetry $\mathrm{G}$ is broken. The embedding tensor will always appear together with the coupling constant $g$ we have introduced in (3.3). The latter could thus be absorbed by rescaling $\Theta_{M}{ }^{\alpha}$, but we will keep it in the following for book-keeping purpose. 
Having introduced the covariant derivatives, the theory should be invariant under the standard combined transformations

$$
\begin{aligned}
\delta \mathcal{V} & =g \Lambda^{M} X_{M} \mathcal{V} \\
\delta A_{\mu}^{M} & =\partial_{\mu} \Lambda^{M}+g A_{\mu}^{N} X_{N P}{ }^{M} \Lambda^{P}=D_{\mu} \Lambda^{M}
\end{aligned}
$$

with local parameter $\Lambda^{M}=\Lambda^{M}(x)$ and $X_{N K}{ }^{M} \equiv \Theta_{N}{ }^{\alpha}\left(t_{\alpha}\right)_{K}{ }^{M}$. This is of course not true for an arbitrary choice of $\Theta$. In particular, consistency requires that the generators (3.4) close into a subalgebra of $\mathfrak{g}$. This in turn translates into a set of non-trivial constraints on the embedding tensor. In the following, we shall work out the complete set of constraints which $\Theta$ must satisfy in order to achieve a theory with local gauge invariance (3.5). As it turns out, there are in general two sets of constraints, a quadratic and a linear one. They can be formulated as G-covariant homogeneous equations in $\Theta$ which allows to construct solutions by purely grouptheoretical methods. Eventually, every solution to this set of constraints will give rise to a consistent Lagrangian with local gauge symmetry (3.5).

The first set of constraints is bilinear in $\Theta$ and very generic. It states that the tensor $\Theta$ is invariant under the action of the generators 3.4 of the local gauge symmetry. Note that $\Theta$ is almost never a G-invariant tensor, as follows already from the different nature of its two indices (except in $D=3$ dimensions, where vector fields transform in the adjoint representation of $\mathrm{G}$ ). Consistency of the gauged theory however requires that $\Theta$ must be invariant under the action of the subgroup $G_{0}$. As this subgroup is precisely defined by projection with $\Theta$, together this leads to a quadratic constraint in $\Theta$ :

$$
\begin{aligned}
0 \stackrel{!}{=} \mathcal{Q}_{P M}{ }^{\alpha} \equiv \delta_{P} \Theta_{M}{ }^{\alpha} & \equiv \Theta_{P}{ }^{\beta} \delta_{\beta} \Theta_{M}{ }^{\alpha} \\
& =\Theta_{P}{ }^{\beta}\left(t_{\beta}\right)_{M}{ }^{N} \Theta_{N}{ }^{\alpha}+\Theta_{P}{ }^{\beta} f_{\beta \gamma}{ }^{\alpha} \Theta_{M}{ }^{\gamma}
\end{aligned}
$$

where we have used the fact that the generators in the adjoint representation are given in terms of the structure constants as $\left(t_{\alpha}\right)_{\beta} \gamma=-f_{\alpha \beta}{ }^{\gamma}$. Contracting this result with a generator $t_{\alpha}$, we obtain the equivalent form

$$
\left[X_{M}, X_{N}\right]=-X_{M N}^{P} X_{P}, \quad \text { with } \quad X_{M N}^{P}=\Theta_{M}^{\alpha}\left(t_{\alpha}\right)_{N}{ }^{P} \text {. }
$$

Hence, the gauge invariance of the embedding tensor in particular implies the closure of the generators (3.4) into an algebra. Let us stress however, that the constraint (3.6) is in general stronger than simple closure: equation 3.7 in particular implies a nontrivial relation upon symmetrization in $(M N)$ (upon which the l.h.s. trivially vanishes, but the r.h.s. does not) which clearly goes beyond closure. Nevertheless this condition turns out to be indispensable and we will come back to it in the next subsection.

Apart from the quadratic constraint (3.6), $\Theta$ must in general satisfy another linear constraint which is implied by supersymmetry. Recall that eventually we wish to construct a theory that does not only possess the local gauge invariance (3.5) but also 
should still be invariant under (a possible deformation of) supersymmetry. This puts further constraints on $\Theta$, whose specific form however will in particular depend on the number of space-time dimensions and supercharges considered. Interestingly enough, in many cases the linear constraint can already be deduced at a much earlier stage by purely bosonic considerations related to consistency of the deformed tensor gauge algebra that we discuss in the next section.

Let us consider as an example the maximal $\mathcal{N}=8, D=4$ theory. Its global symmetry group $\mathrm{G}=\mathrm{E}_{7(7)}$ has 133 generators while the vector fields transform in the fundamental 56 representation. According to its index structure, the embedding tensor $\Theta_{M}{ }^{\alpha}$ thus a priori lives in the tensor product of the fundamental and the adjoint representation, which decomposes according tc ${ }^{2}$

$$
\Theta_{M}^{\alpha}: \quad 56 \otimes 133=56 \oplus 912 \oplus 6480 .
$$

Compatibility of the deformation with supersymmetry can be expressed in an $\mathrm{E}_{7(7)^{-}}$ covariant way and restricts the embedding tensor to the $\mathbf{9 1 2}$ representation in this decomposition [13, 42. I.e. as a matrix $\Theta_{M}{ }^{\alpha}$ has only 912 linearly independent entries. We will sketch the argument in section 3.3 below.

It is interesting to note that in fact also the $\mathbf{5 6}$ part of the embedding tensor in the decomposition (3.8) defines a consistent deformation which however requires simultaneous gauging of the $\mathbb{R}^{+}$on-shell conformal rescaling symmetry of $D=4$ supergravity, see [43] for details. As a result, the corresponding theory no longer admits an action but can be constructed as a supersymmetric deformation of the equations of motion. Deformations of this type have first been constructed in ten space-time dimensions in [44, 45].

As another example, we may consider the half-maximal theory in $D=4$ dimensions, whose scalar fields parametrize the coset space (2.14) with the vector fields transforming in the bifundamental $(\mathbf{2}, \square)$ representation ${ }^{3}$ of $\mathrm{SL}(2) \otimes \mathrm{SO}(6,6+n)$. In analogy to (3.8), the embedding tensor a priori transforms in the tensor product of fundamental and adjoint representation, which decomposes according to

$$
(\mathbf{2}, \square) \otimes((\mathbf{3}, 1)+(\mathbf{1}, \boxminus))=2 \cdot(\mathbf{2}, \square) \oplus(\mathbf{2}, \boxminus) \oplus(\mathbf{2}, \square) \oplus(\mathbf{4}, \square)
$$

Supersymmetry restricts the embedding tensor to $(\mathbf{2}, \square)+(\mathbf{2}, \exists)$, i.e. forbids the last two contributions in (3.9) and poses a linear constraint among the two terms in the $(2, \square)$ representation [47].

In general, the embedding tensor lives within the tensor product

$$
\Theta_{M^{\alpha}}: \quad \mathcal{R}_{\mathrm{v}^{*}} \otimes \mathcal{R}_{\mathrm{adj}}=\mathcal{R}_{\mathrm{v}^{*}} \oplus \ldots
$$

\footnotetext{
${ }^{2}$ All the tensor products and branchings of representations used in these lectures can be found in the appendix of [40] or calculated with the help of the computer algebra package LiE [41].

${ }^{3}$ The box ' $\square$ ' here refers to the vector representation of $\mathrm{SO}(6,6+n)$ and we use the standard Young tableaux notation for the higher irreducible representations.
} 


\begin{tabular}{r||c||c|c||c|}
$D$ & $\mathrm{G}$ & $\mathcal{R}_{\text {adj }}$ & $\mathcal{R}_{\mathrm{v}}$ & $\Theta_{M}{ }^{\alpha}$ \\
\hline 9 & $\mathrm{GL}(2)$ & $\mathbf{1}^{0}+\mathbf{3}^{0}$ & $\mathbf{1}^{-4}+\mathbf{2}^{+3}$ & $\mathbf{2}^{-3}+\mathbf{3}^{+4}$ \\
8 & $\mathrm{SL}(2) \times \mathrm{SL}(3)$ & $(\mathbf{3}, \mathbf{1})+(\mathbf{1}, \mathbf{8})$ & $\left(\mathbf{2}, \mathbf{3}^{\prime}\right)$ & $(\mathbf{2 , 3})+\left(\mathbf{2}, \mathbf{6}^{\prime}\right)$ \\
7 & $\mathrm{SL}(5)$ & $\mathbf{2 4}$ & $\mathbf{1 0}^{\prime}$ & $\mathbf{1 5}+\mathbf{4 0}^{\prime}$ \\
6 & $\mathrm{SO}(5,5)$ & $\mathbf{4 5}$ & $\mathbf{1 6}_{c}$ & $\mathbf{1 4 4}_{c}$ \\
5 & $\mathrm{E}_{6(+6)}$ & $\mathbf{7 8}$ & $\mathbf{2 7 ^ { \prime }}$ & $\mathbf{3 5 1}^{\prime}$ \\
4 & $\mathrm{E}_{7(+7)}$ & $\mathbf{1 3 3}$ & $\mathbf{5 6}$ & $\mathbf{9 1 2}$ \\
3 & $\mathrm{E}_{8(+8)}$ & $\mathbf{2 4 8}$ & $\mathbf{2 4 8}$ & $\mathbf{1}+\mathbf{3 8 7 5}$ \\
2 & $\mathrm{E}_{9(+9)}$ & $\mathcal{R}_{\mathrm{adj}}$ & $\boldsymbol{\Lambda}_{1}$ & $\boldsymbol{\Lambda}_{1 *}$
\end{tabular}

Table 3: Embedding tensor $\Theta_{M}{ }^{\alpha}$ in maximal supergravity. In $D=2$ dimensions, $\mathcal{R}_{\text {adj }}$ and $\boldsymbol{\Lambda}_{1}$ refer to the infinite-dimensional adjoint and basic representation of the affine algebra $\mathrm{E}_{9(9)}=\widehat{\mathrm{E}}_{8(8)}$, respectively.

where by $\mathcal{R}_{\mathrm{v}^{*}}$ we denote the representation dual to the representation $\mathcal{R}_{\mathrm{v}}$ in which the vector fields transform, and the precise form of the r.h.s. depends on the particular group and representations considered. The linear representation constraint then schematically takes the form

$$
\mathbb{P} \Theta=0
$$

and restricts $\Theta$ to some of the representations appearing on the r.h.s. of (3.10). The resulting representations for the embedding tensor in the maximal supergravities are collected in table 3. For the half-maximal theories, the structure is very similar, however the embedding tensor generically contains several different irreducible parts, see e.g. [46, 47. 48, 49].

As we have already mentioned above, the linear representation constraint follows from consistency of the deformation with supersymmetry in first order of $\Theta$, see section 3.3 below. In many cases however, this constraint can already be deduced from purely bosonic considerations and we will see examples of this in the following. E.g. in $D=4$ dimensions, it can be shown [50] that the embedding tensor must in general satisfy the linear constraint

$$
X_{(M N}{ }^{P} \Omega_{K) P}=0
$$

with $X_{M N}{ }^{P}$ from (3.7) and the symplectic matrix $\Omega_{K P}$ from 2.34 , in order to achieve a bosonic Lagrangian with the local symmetry (3.5). This condition does not make any reference to supersymmetry but in particular reproduces the constraints given above for the $\mathcal{N}=8$ and the $\mathcal{N}=4$ theory, respectively $\left.\right|^{4}$

\footnotetext{
${ }^{4}$ It has recently been shown that in $\mathcal{N}=1$ theories this condition may be replaced by an inhomogeneous equation in $\Theta$ in order to cancel the quantum anomaly cubic in $\Theta$ [51]. Here, we restrict the discussion to the classical theories.
} 
To summarize the discussion of this section, any gauging of the theory is entirely encoded in the choice of the embedding tensor $\Theta_{M}{ }^{\alpha}$ which according to (3.4) defines the embedding of the gauge group $\mathrm{G}_{0}$ into the global symmetry group $\mathrm{G}$ and via (3.3) defines the new minimal couplings of vector fields to the remaining matter fields. Consistency of the deformation is expressed by a set of algebraic constraints quadratic (3.6) and linear 3.11) in the embedding tensor, respectively. While it is rather straightforward to verify that the constraints are necessary, it has to be checked case by case (i.e. for the various dimensions $D$ and number of supercharges $N$ ) that indeed they are sufficient to define a consistent gauging. In particular, the action of the gauged theory $\mathcal{L}_{\text {gauged }}$ must be constructed separately in the various space-time dimensions. However once this action has been worked out for generic $\Theta$, all particular gaugings are straightforwardly obtained as specific choices of $\Theta 5^{5}$

The classification of the possible gaugings in a given space-time dimension thus reduces to the analysis of simultaneous solutions of the constraints (3.6) and (3.11). While the latter can be directly solved by working out the explicit projection, the quadratic constraint is in general difficult to solve and does not possess a solution in closed form. The counting of inequivalent gaugings in general remains an unsolved problem. The strategy we will pursue in the following in order to identify the gaugings relevant for the description of particular flux compactifications is the following:

- work out in $D$ dimensions the universal gauged Lagrangian, i.e. the deformation for a generic $\Theta$ solving the constraints 3.6 and $(3.11$.

- identify (exploiting the symmetries related to the higher-dimensional origin) among the components of $\Theta$ those corresponding to particular flux parameters.

- evaluate the quadratic constraint and the general formulas for this particular choice of $\Theta$.

- evaluate the universal formulas for the Lagrangian and in particular the scalar potential for this particular choice of $\Theta$.

- moreover, working out the action of $\mathrm{G}$ on $\Theta$ allows to directly determine the transformation of the flux parameters under the duality group.

We shall illustrate this procedure in section 4 with several explicit examples.

\subsection{Deformed tensor gauge algebra}

We have in the last section defined a gauging in terms of the embedding tensor $\Theta_{M}{ }^{\alpha}$ and started to render the theory invariant under the local symmetry 3.5 by introducing

\footnotetext{
${ }^{5}$ We should mention that for theories with low number of supersymmetries additional quadratic constraints beyond (3.6) may arise, such as the locality of electric and magnetic charges in four dimensions [50]. For the maximal and half-maximal theories discussed here, the quadratic constraint (3.6) is a sufficient condition.
} 
covariant derivatives (3.3). Apart from the minimal couplings induced by (3.3), the field strengths of the vector fields need to be modified in order to capture the nonabelian nature of the new gauge group. This will lead us to a deformation of the higher-rank tensor gauge algebra, intertwining $p$-forms and $(p+1)$-forms. The natural ansatz for the non-abelian field strength of the vector fields is

$$
\mathcal{F}_{\mu \nu}^{M}=\partial_{\mu} A_{\nu}^{M}-\partial_{\nu} A_{\mu}^{M}+g X_{[N P]}^{M} A_{\mu}^{N} A_{\nu}^{P},
$$

but we shall see in the following that this is actually not sufficient.

Let us start from the gauge algebra (3.7)

$$
\left[X_{M}, X_{N}\right]=-X_{M N}^{P} X_{P}
$$

with the "structure constants"

$$
X_{M N}^{P} \equiv \Theta_{M}^{\alpha}\left(t_{\alpha}\right)_{N}^{P} \equiv X_{[M N]}^{P}+Z_{M N}^{P}
$$

where we have introduced the notation $Z^{P}{ }_{M N} \equiv X_{(M N)}{ }^{P}$ for the symmetric part in $X_{M N}{ }^{P}$ which is generically non-vanishing. E.g. for the maximal theories with irreducible embedding tensor $\Theta_{M}{ }^{\alpha}$ (i.e. for $D=4,5,6$, cf. table 3), one may show that there is no gauging for which $Z^{P}{ }_{M N}$ vanishes.

As the l.h.s. of (3.14) is manifestly antisymmetric in $[M N]$, so must be the r.h.s., hence we can deduce that $Z^{P}{ }_{M N}$ vanishes upon contraction with another generator

$$
Z_{M N}^{P} X_{P}=0
$$

as a direct consequence of the quadratic constraint (3.14). The presence of a symmetric part in the "structure constants" $X_{M N}{ }^{P}$ might seem like a bagatelle and simply motivate the definition of the explicitly antisymmetrized $X_{[M N]}{ }^{P}$ as the true structure constants. However, these latter objects fail to satisfy the Jacobi identities:

$$
X_{[M N]}^{P} X_{[Q P]}^{R}+X_{[Q M]}^{P} X_{[N P]}^{R}+X_{[N Q]}^{P} X_{[M P]}^{R}=-Z^{R}{ }_{P[Q} X_{M N]}^{P},
$$

the violation being again proportional to the tensor $Z^{P}{ }_{M N}$. Again the standard Jacobi identity is satisfied upon further contraction with a generator $X_{R}$ as a consequence of (3.16). This is certainly enough for consistency of the algebra (3.14). However, it shows up as a problem in the definition of a suitable covariant field strength tensor which is needed in order to construct a proper covariantization of the abelian kinetic term 2.25), a covariant coupling to the fermionic fields, etc.

Namely, as a consequence of (3.17) the standard non-abelian field strength (3.13) turns out to be not fully covariant. Under the new gauge transformations 3.5 it transforms as

$$
\delta \mathcal{F}_{\mu \nu}^{M}=-g \Lambda^{P} X_{P N}{ }^{M} \mathcal{F}_{\mu \nu}^{N}+2 g Z^{M}{ }_{P Q}\left(\Lambda^{P} \mathcal{F}_{\mu \nu}^{Q}-A_{[\mu}^{P} \delta A_{\nu]}^{Q}\right),
$$


of which only the first term would correspond to a standard homogeneous covariant transformation. Note however that all the unwanted terms appear contracted with the tensor $Z_{P Q}^{M}$ and thus vanish in absence of $Z^{M}{ }_{P Q}$. In particular, together with 3.16 this implies that the combination $\mathcal{F}_{\mu \nu}^{M} X_{M}$, which e.g. shows up in the commutator of covariant derivatives as

$$
\left[D_{\mu}, D_{\nu}\right]=-g \mathcal{F}_{\mu \nu}^{M} X_{M}
$$

is a good covariant object. On the other hand, conceivable covariant kinetic terms constructed from this object such as $\operatorname{Tr}\left[\mathcal{F}_{\mu \nu}^{M} X_{M} \mathcal{F}^{\mu \nu N} X_{N}\right]$ are not smooth deformations of (2.25). A priori it thus remains unclear how to properly covariantize this kinetic term. A similar problem arises in the covariantization of the kinetic terms for the higher-rank $p$-forms 2.26 .

The problem we are facing in this construction is the price to pay for staying Gcovariant. We have chosen a somewhat redundant description of the new gauge group in terms of $n_{\mathrm{v}}$ generators (3.4), whereas in fact in most cases the dimension of the gauge group $\mathrm{G}_{0}$ will be strictly smaller than $n_{\mathrm{v}}$. In other words, the matrix $\Theta_{M}{ }^{\alpha}$ in general does not have maximal rank, thus not all $X_{M}$ are linearly independent. Accordingly, the vector fields $A_{\mu}^{M}$ split into

$$
A_{\mu}^{M} \longrightarrow\left\{\begin{aligned}
A_{\mu}^{m} & \rightarrow \text { transforming in the adjoint of } \mathrm{G}_{0} \\
A_{\mu}^{i} & \rightarrow \text { remaining vectors, transforming in some rep. of } \mathrm{G}_{0}
\end{aligned}\right.
$$

This clearly poses a problem if the $A_{\mu}^{i}$ transform in a non-trivial representation of $\mathrm{G}_{0}$ in which case we cannot write down a consistent gauge theory. As a consequence, w.r.t. this splitting, $Z^{m}{ }_{P Q}$ vanishes whereas $Z^{i}{ }_{P Q} \neq 0$ creates the problems manifest in equation (3.18). This is a generic problem in gauged supergravities which has been encountered ever since such theories have been constructed. Let us briefly discuss how it has been circumvented in some of the early constructions.

- The four-dimensional maximal $\mathrm{SO}(8)$ gauged theory constructed in [1] involves only 28 out of the 56 vector fields that form an irreducible representation of the global symmetry group $\mathrm{E}_{7(7)}$. As discussed in section 2.3 above, only 28 of these vector fields are present in the ungauged action and it is precisely this set which is coupled to the $\mathrm{SO}(8)$ generators. The same situation occurs for the noncompact $\mathrm{SO}(p, q)$ gaugings constructed subsequently in [2]. In other words, the vector fields $A_{\mu}^{i}$ of 3.20 which do not participate in the gauging all live in the magnetic sector and do not appear in the action. It is therefore not a problem that their field strengths are not covariant objects. This distinctive feature of four space-time dimensions has allowed the construction of maximal gaugings without having to address the problem of non-covariance encountered above. However, it strikes back in the presence of magnetic charges and tensor fields [52], and we present its covariant solution below. 
- In five space-time dimensions, the maximal $\mathrm{SO}(6)$ gauging constructed in [3] involves only 15 out of the 27 vector fields, with the 12 remaining ones transforming in a non-trivial representation of $\mathrm{SO}(6)$, implying a non-vanishing $Z^{i}{ }_{P Q}$. The solution found in [3] corresponds to dualizing these unwanted vectors into two-forms (as a particular application of the dualization of $p$-forms (2.29) which upon gauging turn into massive self-dual two-forms, i.e. acquire mass terms of the type [53]

$$
\partial^{\rho} F_{\rho \mu \nu}=e \varepsilon_{\mu \nu \rho \sigma \tau} m F^{\rho \sigma \tau}
$$

with masses $m$ proportional to the inverse coupling constant $g^{-1}$. As a consequence, these forms continue to carry no more than the three degrees of freedom of a massless vector field in five dimensions, consistently keeping the balance of degrees of freedom upon gauging. The Lagrangian of the gauged theory thus carries only 15 vector fields $A_{\mu}^{m}$ with truly covariant field strengths and 12 massive self-dual two-forms. The same mechanism has been successfully applied to other five-dimensional theories, see e.g. [54].

- In seven space-time dimensions the first gaugings of the maximal theory were constructed in [4] and exhibit a somewhat particular situation: the entire set of 10 vector fields present in seven dimensions is needed to gauge an $\mathrm{SO}(5)$ group (or non-compact versions thereof). This is related to the fact, that in seven dimensions the embedding tensor is reducible (cf. table 3); for gaugings defined by an embedding tensor in the $\mathbf{1 5}$ of SL(5) the tensor $Z^{M}{ }_{P Q}$ vanishes identically. For these gaugings however a similar problem shows up upon trying to define a proper covariant field strength for the two-forms. In complete analogy to (3.18), the naive covariantization of their abelian field strengths in general does not transform covariantly. In [4] this problem was circumvented by dualizing all twoforms into three-forms which upon gauging become self-dual massive, analogous to (3.21) thereby conserving the number of degrees of freedom. The Lagrangian of the gauged theory of [4] thus carries only vector fields and massive self-dual three-forms.

In all these cases it has thus eventually been possible to eliminate the extra vector fields $A_{\mu}^{i}$ of 3.20 from the Lagrangian thereby circumventing the problem of the noncovariant field strengths (3.18). This procedure however requires an explicit breaking of the G-covariance: the explicit split 3.20 and thus the field content of the gauged theory depends on the particular gauge group chosen. Furthermore, it remains unclear how to proceed in other space-time dimensions. E.g. a gauging of smaller groups in $D=7$ dimensions cannot be achieved with this construction. Likewise, in even dimensions there is no analogue of the massive self-duality 3.21 which was crucial for the correct balance of degrees of freedom. All this motivates the covariant construction that we shall present in the following, which appears particularly natural in the context of flux compactifications. 
The covariant ansatz makes use of the fact that the non-covariant terms in (3.18) appear projected with the tensor $Z^{M}{ }_{P Q}$ and defines the full covariant field strengths as [14, 15]

$$
\mathcal{H}_{\mu \nu}^{M}=\mathcal{F}_{\mu \nu}^{M}+g Z^{M}{ }_{P Q} B_{\mu \nu}^{P Q},
$$

upon the introduction of two-form tensor fields of the type $B_{\mu \nu}^{M N}=B_{[\mu \nu]}^{(M N)}$. The non-covariant terms in (3.18) can then be absorbed by postulating the corresponding transformation laws for the two-form fields. Explicitly, the new field strength $\mathcal{H}_{\mu \nu}^{M}$ transforms covariantly under the combined set of gauge transformations

$$
\begin{aligned}
\delta A_{\mu}^{M} & =D_{\mu} \Lambda^{M}-g Z_{P Q}^{M} \Xi_{\mu}^{P Q}, \\
\delta B_{\mu \nu}^{M N} & =2 D_{[\mu} \Xi_{\nu]}^{M N}-2 \Lambda^{(M} \mathcal{H}_{\mu \nu}^{N)}+2 A_{[\mu}^{(M} \delta A_{\nu]}^{N)},
\end{aligned}
$$

where $\Xi_{\mu}^{M N}$ labels the tensor gauge transformations associated with the two-forms. A non-vanishing tensor $Z^{M}{ }_{P Q}$ thus induces a Stückelberg-type coupling between vector fields and antisymmetric two-forms, as is familiar from massive deformations of supergravities, e.g. [55]. It is the strength of the covariant formalism to treat all possible deformations (gauged and massive supergravities) on the same footing.

Of course, the two-forms $B_{\mu \nu}^{M N}$ introduced in 3.22 cannot simply be added to the fields of the theory, as the number of degrees of freedom is in general carefully balanced by supersymmetry. Rather, these must be (a subset of) the two-forms that are already present in the ungauged supergravity. Their index structure in $(3.22)$ shows that they generically appear in a representation of $G$ that is contained in the symmetric tensor product $\left(\mathcal{R}_{\mathrm{v}} \otimes \mathcal{R}_{\mathrm{v}}\right)_{\text {sym }}$. Their precise representation can be inferred from inspection of the tensor $Z^{M}{ }_{P Q}$ under which they appear. In turn, this severely constrains the tensor $Z^{M}{ }_{P Q}$ which in its indices $(P Q)$ should project only onto those representations filled by the two-forms in the ungauged theory. As $Z$ is a function of the embedding tensor $\Theta$, this eventually leads to a linear representation constraint of the type (3.11) on $\Theta$.

As an example, let us consider the case of $D=4$ dimensions wher $\oplus^{6}$

$$
\begin{aligned}
Z^{K}{ }_{M N} & =X_{(M N)}{ }^{K} \\
& =\frac{1}{2} \Theta_{M}{ }^{\alpha}\left(t_{\alpha}\right)_{N}{ }^{K}+\frac{1}{2} \Theta_{N}{ }^{\alpha}\left(t_{\alpha}\right)_{M}{ }^{K} \\
& =-\frac{1}{2} \Theta^{K \alpha}\left(t_{\alpha}\right)_{M N}+\frac{3}{2} X_{(M N L)} \Omega^{K L},
\end{aligned}
$$

where we have made use of the fact that following the discussion of section 2.3, in $D=4$ dimensions the symmetry generators are embedded into the symplectic group, i.e. $\left(t_{\alpha}\right)_{[M N]}=0$. Plugging this expression into the covariant field strength 3.22 shows

\footnotetext{
${ }^{6}$ In these formulas we have been raising and lowering indices $M, N$ with the symplectic matrix $\Omega^{M N}$ and north-west south-east conventions, i.e. $\Theta^{M \alpha}=\Omega^{M N} \Theta_{N}{ }^{\alpha}$, etc.
} 
that if the embedding tensor satisfies the linear constraint $X_{(M N L)}=0$ of $(3.12)$, the two-forms in (3.22) appear always under the particular projection

$$
Z^{K}{ }_{M N} B_{\mu \nu}^{M N} \equiv-\frac{1}{2} \Theta^{K \alpha} B_{\mu \nu \alpha}, \quad \text { with } \quad B_{\mu \nu \alpha}=\left(t_{\alpha}\right)_{M N} B_{\mu \nu}^{M N}
$$

They thus can be labeled by indices in the adjoint representation of the global symmetry group G. In other words, out of the two-forms $B_{\mu \nu}^{M N}$ in the symmetric tensor product 7

$$
\left(\mathcal{R}_{\mathrm{v}} \otimes \mathcal{R}_{\mathrm{v}}\right)_{\mathrm{sym}}=\mathcal{R}_{\mathrm{adj}} \oplus \ldots
$$

only those transforming in the adjoint representation $\mathcal{R}_{\text {adj }}$ are involved in the gauging. This is precisely in accordance with the fact that two-forms in four dimensions are dual to the scalar field isometries as a consequence of the on-shell duality (2.32) and thus transform in the adjoint representation of G. The argument shows the need for the linear representation constraint $(3.12)$ in $D=4$ dimensions from purely bosonic considerations, ensuring that the gauge algebra can be rendered consistent by adding precisely the two-forms $B_{\alpha}$ that we have at our disposal. On the other hand, an embedding tensor satisfying $X_{(M N L)} \neq 0$ would require the coupling of more two-forms (in the full symmetric tensor product (3.26) ) for consistency of the tensor gauge algebra, in contradiction with the given field content.

To summarize, we have seen in this section that a general gauging not only requires covariant derivatives (3.3) but also Stückelberg-type couplings between vector fields and two-form tensors in order to define the covariant field strengths (3.22). Consistency of the tensor gauge algebra poses linear representation constraints (3.11) on the embedding tensor, i.e. restricts the possible gaugings. In fact, the presence of two-form tensor fields in the effective actions precisely fits with what is observed in explicit flux compactifications, e.g. the massive two-form field appearing in particular flux compactifications on Calabi-Yau manifolds [16].

Let us close this section with a few remarks on the vector/tensor gauge transformations 3.23

- Strictly speaking, the variation of $B_{\mu \nu}^{M N}$ as it stands in $\sqrt{3.23}$ is only exact under projection with the tensor $Z^{P}{ }_{M N}$ - this is for instance of importance when verifying closure of the gauge algebra (3.27) below.

- The gauge transformations 3.23 close into the algebra

$$
\begin{aligned}
{\left[\delta\left(\Lambda_{1}\right), \delta\left(\Lambda_{2}\right)\right]=} & \delta(\Lambda)+\delta(\Xi) \\
\text { with } \quad & \Lambda^{M}=g X_{[N P]}^{M} \Lambda_{1}^{N} \Lambda_{2}^{P}, \\
& \Xi_{\mu}^{M N}=\Lambda_{1}^{(M} D_{\mu} \Lambda_{2}^{N)}-\Lambda_{2}^{(M} D_{\mu} \Lambda_{1}^{N)}
\end{aligned}
$$

\footnotetext{
${ }^{7}$ The fact that the adjoint representation of $\mathrm{G}$ appears in the symmetric tensor product of the vector field representation is just another way of expressing the fact that in $D=4$ dimensions the group $\mathrm{G}$ is embedded into $\operatorname{Sp}(m, m)$ as discussed in section 2.3
} 
showing once more the need of introducing the extra Stückelberg shift $\Xi_{\mu}^{M N}$ on the vector fields in (3.23) in order to close the gauge algebra. In the presence of higher-rank tensor fields, the r.h.s. of 3.27) in general contains also the corresponding higher-rank tensor gauge transformations, whose action on the vector fields is trivial.

- Naively, one might have expected the standard homogeneous transformation behavior $\delta B^{M N}=-2 g \Lambda^{K} X_{K L}{ }^{(M} B^{N) L}$ of the two-forms under gauge transformations, rather than the covariant $\Lambda^{(M} \mathcal{H}^{N)}$ term in 3.23 . However, the latter contains a contribution $-2 g \Lambda^{(M} Z^{N)}{ }_{P Q} B^{P Q}$ which comes close to the above homogenous term. The discrepancy precisely vanishes under projection with $Z^{R}{ }_{M N}$ as a consequence of the identity

$$
Z^{R}{ }_{M P} X_{S Q}{ }^{M}+Z^{R}{ }_{M Q} X_{S P}{ }^{M}-2 Z^{R}{ }_{M S} Z^{M}{ }_{P Q}=0
$$

which in turn follows as a consequence of the quadratic constraint (3.14). Under projection thus one recovers from (3.23) the standard homogenous transformation behavior. Most importantly, the full field strengths $\mathcal{H}^{M}$ transform covariantly under the gauge transformations 3.23).

- The full covariant field strength $(3.22)$ no longer satisfies the standard Bianchi identies, but rather its deformed version

$$
D_{[\mu} \mathcal{H}_{\nu \rho]}^{M}=\frac{1}{3} g Z^{M}{ }_{P Q} \mathcal{H}_{\mu \nu \rho}^{P Q}
$$

where $\mathcal{H}_{\mu \nu \rho}^{P Q}$ denotes the covariant field strength of the two-forms.

- The combined transformation

$$
\Xi_{\mu}^{M N}=D_{\mu} \xi^{M N}, \quad \Lambda^{M}=g Z_{P Q}^{M} \xi^{P Q}
$$

has no effect on the gauge fields $A_{\mu}^{M}, B_{\mu \nu}^{M N}$ (the latter again under projection with $Z_{M N}^{P}$ ). This is the proper non-abelian generalization of the standard tensor gauge redundancy.

- The structure we have presented here for vector fields and two-forms extends to the full hierarchy of higher-rank $p$-forms. In particular, the full set of vector/tensor gauge transformations takes the form (schematically)

$$
\begin{aligned}
\delta \mathcal{V} & =g \Theta \Lambda \mathcal{V} \\
\delta A_{\mu} & =D_{\mu} \Lambda-g \Theta \Xi_{\mu} \\
\delta B_{\mu \nu} & =2 D_{[\mu} \Xi_{\nu]}+\ldots-g \Theta \Phi_{\mu \nu}, \\
\delta C_{\mu \nu \rho} & =3 D_{[\mu} \Phi_{\nu \rho]}+\ldots-g \Theta \Sigma_{\mu \nu \rho} \\
\text { etc. } &
\end{aligned}
$$


where we have ommitted all G-indices. This shows the non-trivial entanglement between $p$-forms and ( $p-1)$-forms via Stückelberg terms induced by a generic gauging (i.e. a generic tensor $\Theta$ ). We have schematically denoted all the intertwining tensors by $\Theta$ as they are uniquely defined in terms of the embedding tensor, while their precise index structure takes care of the different representations in which the $p$-forms transform (with $Z^{K}{ }_{M N}$ from 3.15 as the lowest explicit intertwining tensor). The representation content of the embedding tensor is determined from the linear constraint (3.11) which we have seen to follow from consistency of the tensor gauge algebra on the lowest-rank tensor fields. Remarkably, a closer analysis of the higher-rank tensor gauge transformations 3.31) then allows to determine the representation content of all higher-rank $p$-forms of the theory, see [15, 56]. For the maximal supergravities, with the embedding tensor given in table 3, this reproduces the entire field content of these theories, including the non-propagating $(D-1)$ and $D$-forms. In particular, it gives agreement with the predictions obtained from analyzing the branchings of the infinite-dimensional representations of the underlying very extended Kac-Moody algebras, [25, 26, 49, 57].

The task in the following will be to put all these structures on the level of the Lagrangian.

\subsection{The Lagrangian}

In this section we will describe how to obtain the Lagrangian that is compatible with the new local symmetry (3.23) as a deformation of the Lagrangian of the ungauged theory. Given the algebraic framework we have set up in the last two sections, the first step obviously consists of covariantizing all derivatives according to (3.3) and to replace

the abelian field strengths by the full covariant ones $(3.22)$ and their analogues for the higher-rank $p$-forms. It is slightly more tedious but straightforward to also covariantize the topological terms present in the ungauged theory.

We have seen in the last section, that the deformation leads to an entanglement of the $p$-forms and the $(p+1)$-forms via the corresponding field strengths. As a consequence, the covariantized Lagrangian will carry forms of higher degree than the ungauged one. E.g. in $D=4,5$ dimensions, the gauged theory generically carries 2 -forms, in $D=6,7$ dimensions the gaugings carry 3 -forms, etc., see table 4 (cf. in contrast table 2 for the ungauged theories). Moreover, since the construction of the deformation is manifestly G-covariant, the gauged theories in even dimensions generically carry the full G-representation of forms rather that only the electric half.

A priori, the presence of these extra fields in the gauged theory might pose a formidable obstacle to the construction: as these fields were not present in the ungauged theory, they do not possess kinetic terms but instead only appear as corrections to lower-rank field strengths and topological terms upon covariantization. This might lead to weird if not inconsistent additional field equations. Instead, somewhat miraculously, 


\begin{tabular}{r|c||c|c|c|c|}
$D$ & $\mathrm{G}$ & 1 & 2 & 3 & 4 \\
\hline 9 & $\mathrm{GL}(2)$ & $\mathbf{1}^{-4}+\mathbf{2}^{+3}$ & $\mathbf{2}^{-1}$ & $\mathbf{1}^{+2}$ & $\mathbf{1}^{-2}$ \\
8 & $\mathrm{SL}(2) \times \mathrm{SL}(3)$ & $\left(\mathbf{2}, \mathbf{3}^{\prime}\right)$ & $(\mathbf{1}, \mathbf{3})$ & $(\mathbf{2}, \mathbf{1})$ & $\left(\mathbf{1}, \mathbf{3}^{\prime}\right)$ \\
7 & $\mathrm{SL}(5)$ & $\mathbf{1 0}^{\prime}$ & $\mathbf{5}$ & $\mathbf{5}^{\prime}$ & \\
6 & $\mathrm{SO}(5,5)$ & $\mathbf{1 6}_{c}$ & $\mathbf{1 0}$ & $\mathbf{1 6}_{s}$ & \\
5 & $\mathrm{E}_{6(6)}$ & $\mathbf{2 7}^{\prime}$ & $\mathbf{2 7}$ & & \\
4 & $\mathrm{E}_{7(7)}$ & $\mathbf{5 6}$ & $\mathbf{1 3 3}$ & & \\
3 & $\mathrm{E}_{8(8)}$ & $\mathbf{2 4 8}$ & & &
\end{tabular}

Table 4: The $p$-forms $(p \geq 1)$ entering the Lagrangian of gauged maximal supergravity.

it turns out that the various contributions from kinetic and topological terms precisely combine into first-order field equations for the additional fields. This reflects the fact that these fields do not constitute additional degrees of freedom but are the on-shell duals of the fields of the ungauged theory. From this perspective, remarkably, this reasoning gives a purely bosonic argument for the appearance of the topological terms in the ungauged theory: it is their covariantization that renders the field equations in the gauged theory consistent 8 In other words, in absence of the standard topological term (whose presence is usually deduced from supersymmetry) the bosonic theories would not allow for generic deformations.

Let us discuss as an example the theories in $D=4$ space-time dimensions. The general gauging is defined by an embedding tensor $\Theta_{M}{ }^{\alpha}$ giving rise to covariant derivatives 3.3 which in general involve all vector fields

$$
D_{\mu} \equiv \partial_{\mu}-g A_{\mu}^{M} \Theta_{M}{ }^{\alpha} t_{\alpha}=\partial_{\mu}-g A_{\mu}^{\Lambda} \Theta_{\Lambda}{ }^{\alpha} t_{\alpha}-g A_{\mu \Lambda} \Theta^{\Lambda \alpha} t_{\alpha}
$$

where according to the discussion of section 2.3 we have split the $2 m$ vector fields into the $m$ electric ones $A_{\mu}^{\Lambda}$ and their magnetic duals. Only the former ones appear in the ungauged theory. While at first sight it may seem unnatural to include the magnetic vector fields in the general connection, this is in fact indispensable in order to achieve a duality covariant description of flux compactifications. Recall that upon different compactification from higher dimensions one usually ends up in different symplectic frames in four dimensions. I.e. depending on the higher-dimensional context, the effective four-dimensional theory might carry different selections of $m$ electric vector fields among the $2 m$ gauge fields. Only after exchanging some electric versus magnetic vector fields, one may be able to pin down the equivalence/duality between different compactifications. This shows that a restriction to electric vector fields in 3.32 might miss certain effective theories which correspond to standard electric gaugings in another symplectic frame. On the other hand, with all components of $\Theta_{M}{ }^{\alpha}$ present in (3.32) it is straightforward to identify the action of the duality group onto the various flux

\footnotetext{
${ }^{8}$ A notable exception is the three-dimensional theory, whose ungauged version does not carry any topological term, such that a gauge invariant Chern-Simons term for the vector fields must be added to the gauged theory, in order to produce sensible field equations.
} 
parameters in different compactifications. We shall see this in more detail in the last section.

The appearance of the $A_{\mu \Lambda}$ in the covariant derivatives (3.32) could lead to the problems discussed above: as new fields they seem to appear in the role of Lagrange multipliers that would imply some devastating field equations. However, the above discussed mechanism comes to the rescue: gauge invariance of the Lagrangian (2.36), also requires the introduction of additional topological terms of the form [50]

$$
\mathcal{L}_{\text {top }} \propto \varepsilon^{\mu \nu \sigma \tau}\left(g \Theta^{\Lambda \alpha} \partial_{\mu} A_{\nu \Lambda} B_{\sigma \tau \alpha}+\frac{1}{8} g^{2} \Theta^{\Lambda \alpha} \Theta_{\Lambda}{ }^{\beta} B_{\mu \nu \alpha} B_{\sigma \tau \beta}+\ldots\right)
$$

Together, it follows from variation of $B_{\mu \alpha}$ that

$$
g \Theta^{\Lambda \alpha}\left(\mathcal{H}_{\mu \nu \Lambda}+e \varepsilon_{\mu \nu \sigma \tau} \frac{\delta \mathcal{L}_{\text {kin }}}{\delta \mathcal{H}_{\sigma \tau} \Lambda}\right)=0
$$

which precisely reproduces the covariant version of the duality equation (2.38). Likewise, variation w.r.t. the magnetic vector fields induces the duality equation 2.32 between scalars and two-forms. Note that for the gauged theory, the duality equations arise as true field equations, however projected with the matrix $\Theta^{\Lambda \alpha}$. In particular, in the limit $g \rightarrow 0$, all dual fields disappear from the Lagrangian and equation (3.34) consistently decouples. This is different from the democratic formulation of supergravities, in which the dual fields are introduced already in the ungauged action and the duality relations 2.38), etc. must be supplied by hand.

Summarizing, we have succeeded in finding a deformation of the original ungauged Lagrangian of supergravity that is compatible with the algebraic structures induced by the new local gauge group and encoded in the embedding tensor $\Theta_{M}{ }^{\alpha}$ as presented in the last sections. Details of the construction may differ in the various space-time dimensions and can be found in the literature for several examples, see e.g. 12, 14, 50, 58, 47, 59, 60, 61, 62, 42, 63] 9. The fact that the deformation has been described in a manifestly G-covariant way has another appealing consequence: in even dimensions - where $\mathrm{G}$ is realized only on-shell - this construction can accommodate gaugings of subgroups $\mathrm{G}_{0}$ of $\mathrm{G}$ that are not among the off-shell symmetries of the ungauged Lagrangian!

Now, that we have constructed a gauge invariant Lagrangian, we may take the next step and check if the deformation is further compatible with local supersymmetry. As it stands, the deformed Lagrangian is no longer invariant under supersymmetry due to the extra contributions that arise from variation of the vector fields in the covariant derivatives, and from the deformation of Bianchi identities (3.29), etc. in the gauged theories. Supersymmetry can be restored applying the standard Noether procedure [1].

\footnotetext{
${ }^{9}$ In dimensions $D=8$ and $D=9$, gauged supergravities have been classified and constructed independently, based on the different compactification manifolds [64, 65. While the nine-dimensional case is in exact agreement with the form of the embedding tensor of table 3 , the compactifications to eight dimensions seem to reproduce only part of the possible components of the corresponding embedding tensor.
} 
In linear order of the deformation parameters $\Theta$, the unwanted contributions can be cancelled by introducing particular fermionic mass terms of the type (schematically)

$$
\mathcal{L}_{\text {ferm-mass }}=g\left(\bar{\psi}^{i} A_{i j} \psi^{j}+\bar{\chi}^{A} B_{A i} \psi^{i}+\bar{\chi}^{A} C_{A B} \chi^{B}\right)+\text { h.c. },
$$

where by $\psi^{i}$ and $\chi^{A}$ we denote gravitinos and spin-1/2 fermions, respectively, with the indices $i$ and $A$ labeling the respective K-representations, and where we have suppressed all space-time indices and $\gamma$-matrices ${ }^{10}$ The tensors $A_{i j}, B_{A i}$, and $C_{A B}$ may depend on the scalar fields, and inherit their symmetry properties from their precise appearance in (3.35). Under the action 2.5) of $\mathrm{K}$ they should transform in the proper representations such that (3.35) is K-invariant. Together with the fact that these tensors must be defined in terms of the embedding tensor $\Theta_{M}{ }^{\alpha}$ that encodes the deformation, their transformation properties entirely fix the form of these tensors. Specifically, they are constructed from the so-called $T$-tensor defined by

$$
T_{\underline{N}}^{\underline{\beta}} \equiv \Theta_{M}^{\alpha} \mathcal{V}^{M}{ }_{N} \mathcal{V}_{\alpha} \underline{\beta},
$$

as the embedding tensor dressed with the scalar group matrix $\mathcal{V}$ evaluated in the fundamental and the adjoint representation of $\mathrm{G}$, respectively. This object has first appeared in the $\mathrm{SO}(8)$ gauging in $D=4$ dimensions [1]. In contrast to the constant $\Theta_{M}{ }^{\alpha}$, the $T$-tensor depends non-trivially on the scalar fields. As it is obtained from the embedding tensor by a finite $\mathrm{G}$ transformation, it lives in the same G-representation as $\Theta$, i.e. it inherits from $\Theta$ the linear constraint 3.11

$$
\mathbb{P} T=0,
$$

which now holds for any value of the scalar fields on which $T$ depends. Under $\mathrm{K}$ this tensor contains various irreducible parts, obtained by decomposing the G-representation of $\Theta$ (cf. table 3) under the compact subgroup K. These K-irreducible parts can precisely be identified with the fermionic mass tensors in 3.35).

For example in $D=4, \mathcal{N}=8$, under $\mathrm{K}=\mathrm{SU}(8)$ the embedding tensor breaks into

$$
\Theta_{M}^{\alpha} \longrightarrow T_{\underline{M}}^{\underline{\alpha}} \rightarrow\left(A^{i j}, A_{i j}, B^{A i}, B_{A i}\right)
$$

according to the decomposition $912 \rightarrow \mathbf{3 6}+\overline{\mathbf{3 6}}+\mathbf{4 2 0}+\overline{\mathbf{4 2 0}}$, from which the fermionic mass tensors are built. For the explicit formulas of the tensors $\left(A^{i j}, A_{i j}, B^{A i}, B_{A i}\right)$ in terms of a general $\Theta$, we refer to [42].

Turning the argument around, this shows the origin of the linear representation constraint from supersymmetry. The supersymmetry-violating terms in the Lagrangian which are proportional to the embedding tensor, e.g. as (schematically)

$$
F_{\mu \nu}^{M} \Theta_{M}^{\alpha} \mathcal{V}_{\alpha}^{\underline{\beta}}(\bar{\epsilon} \psi)_{\underline{\beta}}=\left(F_{\mu \nu}^{M} \mathcal{V}_{M}^{\underline{N}}\right) T_{\underline{N}} \underline{\beta}(\bar{\epsilon} \psi)_{\underline{\beta}}
$$

\footnotetext{
${ }^{10}$ Our treatment of fermions will remain somewhat schematic in this section as we are trying to give a discussion for arbitrary theories, whereas e.g. their symmetry and hermiticity properties certainly depend on the number $D$ of space-time dimensions and $N$ of supersymmetries, see e.g. [66, 28, 30] for a discussion of spinor fields in various dimensions.
} 
can be cancelled by the variation of the additional fermionic mass terms (3.35) if and only if the tensor $T_{\underline{N}} \underline{\beta}$ can be built from the representations of proper fermionic mass tensors. In $D=4, \mathcal{N}=8$, the possible fermionic mass tensors fall into $\mathrm{SU}(8)$ representations

$$
\begin{aligned}
(\bar{\psi} \psi): & (\mathbf{8} \otimes \mathbf{8})_{\mathrm{sym}}=\mathbf{3 6} \\
(\bar{\psi} \chi): & (\mathbf{8} \otimes \overline{\mathbf{5 6}})=\mathbf{2 8}+\overline{\mathbf{4 2 0}} \\
(\bar{\chi} \chi): & (\overline{\mathbf{5 6}} \otimes \overline{\mathbf{5 6}})_{\mathrm{sym}}=\mathbf{4 2 0}+\overline{\mathbf{1 1 7 6}}
\end{aligned}
$$

and their hermitean conjugates. Comparing this to the possible representation content of a generic embedding tensor $\Theta_{M}{ }^{\alpha}$ from 3.8

$$
\begin{aligned}
56 & \rightarrow 28+\text { h.c. } \\
912 & \rightarrow 36+420+\text { h.c. } \\
6480 & \rightarrow 28+420+1280+1512+\text { h.c. },
\end{aligned}
$$

shows that an embedding tensor $\Theta$ in the $\mathbf{6 4 8 0}$ gives rise to terms of the type 3.39 with a part of $T_{\underline{N}} \underline{\beta}$ in the $1280+\mathbf{1 5 1 2}$ which cannot be cancelled by fermionic mass terms 3.40 . This is the underlying reason why supersymmetry requires the linear constraint (3.11) and forbids a $\Theta$ in the $\mathbf{6 4 8 0}$. Similarly, a $\Theta$ in the $\mathbf{5 6}$ is ruled out by supersymmetry: although a $\mathbf{2 8}$ appears in $(\bar{\psi} \chi)$, a closer check shows that its absence in $(\bar{\psi} \psi)$ forbids this representation in the embedding tensor. Moreover, 3.40 shows that in this theory the mass tensor $C_{A B}$ of the spin- $1 / 2$ fermions is in fact obtained from the mixed mass tensor $B^{A i}$ - as there is only a single 420 contribution within the 912. Indeed, this was first discovered in the $\mathrm{SO}(8)$ gauging of [1].

Let us recall that in the previous sections we have found linear representation constraints on the embedding tensor from purely bosonic considerations - consistency of the deformed $p$-form tensor hierarchy. It is remarkable and somewhat surprising that supersymmetry appears to impose precisely the same linear constraint on the possible deformations such that no further restriction descends from compatibility with supersymmetry. 11

If the embedding tensor satisfies the linear representation constraint, the additional fermionic mass terms 3.35) are precisely sufficient to cancel all supersymmetryviolating terms in linear order of $\Theta$ if simultaneously the fermionic supersymmetry transformations are modified according to (schematically)

$$
\delta \psi^{i}=\delta_{0} \psi^{i}-g A^{i j} \epsilon_{j}, \quad \delta \chi^{A}=\delta_{0} \chi^{A}-g B^{A i} \epsilon_{i},
$$

where $\delta_{0}$ denotes the (properly covariantized) supersymmetry transformations of the ungauged theory. The reason for the additional fermion-shifts in 3.42 is to cancel the $D_{\mu} \epsilon$ contributions descending from 3.35 .

\footnotetext{
${ }^{11}$ Again, a notable exception is the three-dimensional theory, where supersymmetry imposes linear constraints on the embedding tensor that do not already follow from consistency of the bosonic Lagrangian 67.
} 
Finally, supersymmetry in second order $g^{2}$ of the deformation requires the addition of a scalar potential, which is schematically of the form

$$
\mathcal{L}_{\text {pot }}=-e V=-e g^{2}\left(B^{A i} B_{A i}-A^{i j} A_{i j}\right),
$$

in terms of the fermionic mass tensors, in order to cancel the $g^{2}$ contributions descending from the action of 3.42 on (3.35). It is characteristic for supergravity theories that - in contrast to globally supersymmetric theories - the scalar potential is in general not positive definite, but may in particular support $\mathrm{dS}$ and $\mathrm{AdS}$ vacua. For particular gaugings, i.e. particular choices of $\Theta$, despite its appearance of $(3.43)$, the potential may still be positive definite.

Consistent cancellation of all supersymmetry variations in order $g^{2}$ typically requires a number of nontrivial algebraic identities to be satisfied by the fermionic mass tensors $A_{i j}, B_{A i}$, and $C_{A B}$. In particular, one needs the traceless condition

$$
g^{2}\left(B^{A i} B_{A j}-A^{i k} A_{j k}\right) \equiv \frac{1}{N} \delta_{j}^{i} V
$$

with $N$ the number of supercharges and the scalar potential $V$ from (3.43) - often referred to as a supersymmetric Ward identity. As this is a condition which is bilinear in the embedding tensor, the only way it can be satisfied without imposing further constraints on the gauging is as a consequence of the quadratic constraint (3.6). Indeed, in all dimensions, (3.44) and analogous relations can be derived from (3.6) upon dressing the latter with the scalar matrix $\mathcal{V}$ and breaking it into its K-irreducible parts.

It is sometimes convenient to express the scalar potential directly in terms of the embedding tensor rather than going through the process of computing the fermionic mass tensors. E.g. for the maximal $\mathcal{N}=8$ theory in $D=4$ dimensions, the potential takes the equivalent form 42 ]

$$
V=g^{2}\left(X_{M N}{ }^{R} X_{P Q}{ }^{S} \mathcal{M}^{M P} \mathcal{M}^{N Q} \mathcal{M}_{R S}+7 X_{M N}{ }^{Q} X_{P Q}{ }^{N} \mathcal{M}^{M P}\right),
$$

with $X_{M N}{ }^{K}$ defined in (3.7) as a function of the embedding tensor, and the positive definite scalar matrices $\mathcal{M}_{M N}$ defined in (2.15). This provides a universal and very compact form for the scalar potential obtained in generic flux compactifications. Depending on the particular fluxes present in the compactification, different blocks of the embedding tensor will be non-vanishing and shape the dependence of $V$ on the scalar fields contained in $\mathcal{M}_{M N}$. We will come back to this in section 4 .

In general, the scalar potential can always be cast into the form

$$
V=g^{2} \mathrm{~V}_{\alpha \beta}^{M N} \Theta_{M}^{\alpha} \Theta_{N}^{\beta}
$$

in terms of the embedding tensor and a scalar dependent matrix $\mathrm{V}^{M N}{ }_{\alpha \beta}$ which e.g. for the maximal $N=8$ theory can be extracted from (3.45). Interestingly, this matrix shows up in the analogue of the duality relations $(2.29)$ for the $(D-1)$ forms of the theory. These non-propagating forms, whose field content can e.g. be deduced from 
the supersymmetry algebra of the ungauged theory [68, 57, 56] in general transform in the representation dual to the embedding tensor, i.e. carry indices of the type $C^{M}{ }_{\alpha}$. Whereas these forms are usually set to zero in the ungauged theory, their G-covariant equations of motion can be integrated to [56]

$$
\partial_{\left[\mu_{1}\right.}\left(C^{M}{ }_{\alpha}\right)_{\left.\mu_{2} \ldots \mu_{D}\right]}+\cdots=e \varepsilon_{\mu_{1} \ldots \mu_{D}} \mathrm{~V}^{M N}{ }_{\alpha \beta} \vartheta_{N}^{\beta}
$$

with integration constants $\vartheta_{N}{ }^{\beta}$ and the dots representing possible Chern-Simons contributions to the field strength. Non-vanishing integration constants in this equation precisely induce the gauged theory with the identification $\vartheta_{N}{ }^{\beta} \equiv \Theta_{N}{ }^{\beta}$.

\section{Flux Compactifications - Examples}

In this final section we will work out a few explicit examples of four-dimensional gauged supergravities associated to particular flux compactifications along the lines discussed at the end of section 3.1. The maximal and half-maximal supergravities are in particular relevant for flux compactifications on tori (and their orientifolds) and we will mainly consider the torus compactifications from M-theory and the IIA/IIB theories.

The simplest flux compactifications refer to compactifications with non-trivial values

$$
\int_{\Sigma} \mathcal{F}^{(p)}=\mathcal{C}_{\Sigma}
$$

of $p$-form field strengths $\mathcal{F}$ along non-trivial cycles $\Sigma$ of the internal manifold. The constants $\mathcal{C}_{\Sigma}$ can be considered as deformation parameters and as such be identified within the components of the embedding tensor $\Theta_{M}{ }^{\alpha}$ introduced above. In the following, we will mainly consider compactifications on tori $T^{n}$, where the non-trivial cycles are products of circles and thus labeled by indices along the directions of the torus.

\subsection{Higher-dimensional origin of symmetries}

A crucial role in the covariant construction of gaugings was played by the underlying global symmetry groups $G$ of the ungauged theories, given in table 1 . In order to work out the gaugings which correspond to the effective theories of particular flux compactifications, it will thus be important to first understand the higher-dimensional origin of these symmetry groups. Recall, that the ungauged maximal and the halfmaximal theories arise from reduction of eleven- and ten-dimensional supergravity, respectively, on an $N$-torus, with the global symmetry groups given by exceptional and the orthogonal series $\mathrm{E}_{N(N)}$ and $\mathrm{SO}(N, N)$, respectively. In both cases, the maximal $\mathrm{GL}(N)$ subgroups have a relatively simple higher-dimensional interpretation related to the geometry of the $N$-torus, while the remaining part of the groups is related to higher-dimensional tensor fields. 
Let us first consider the reduction of pure gravity from $(D+N)$ dimensions down to $D$ dimensions. With coordinates splitting according to $x^{M} \rightarrow\left(x^{\mu}, y^{m}\right), \mu=0, \ldots, D-$ $1 ; m=1, \ldots N$, and similarly for the flat indices $A \rightarrow(\alpha, a)$, the reduction ansatz for the vielbein on an $N$-torus is given by

$$
E_{M}^{A}=\left(\begin{array}{cc}
\mathrm{e}^{\kappa \phi} e_{\mu}{ }^{\alpha} & \mathrm{e}^{\phi / N} V_{m}{ }^{a} B_{\mu}^{m} \\
0 & \mathrm{e}^{\phi / N} V_{m}{ }^{a}
\end{array}\right),
$$

with all components depending only on the coordinates $x^{\mu}$. The matrix $V_{m}{ }^{a}$ is normalized by $\operatorname{det} V=1$, and $\kappa=\frac{1}{2-D}$ is chosen such that the lower dimensional action appears again in the Einstein frame. The $D$-dimensionsal theory thus carries a vielbein, $N$ vector fields and $N^{2}$ scalar fields. The ansatz (4.2) preserves an $\mathrm{SO}(1, D-1) \times \mathrm{SO}(N)$ subgroup of the original Lorentz group. The second factor can be used to remove $\frac{1}{2} N(N-1)$ of the components in $V_{m}{ }^{a}$ by virtue of

$$
\delta V_{m}{ }^{a}=V_{m}{ }^{b} h^{a}{ }_{b}, \quad h \in \mathfrak{s o}(N),
$$

leaving $\frac{1}{2} N(N+1)$ physical scalars in the reduced theory.

The diffeomorphism symmetries $\xi^{M}$ of the $(D+N)$-dimensional theory induce different symmetries in the reduced theory. While diffeomorphisms $\xi^{\mu}(x)$ induce $D$ dimensional diffeomorphisms, it is easy to check that the diffeomorphisms $\xi^{m}(x)$ along the compactified directions induce abelian gauge transformations for the Kaluza-Klein vector fields

$$
\delta B_{\mu}^{m}=\partial_{\mu} \xi^{m}
$$

Moreover, diffeomorphisms linear in the $N$ compactified coordinates, $\xi^{m}=-\Lambda_{n}^{m} y^{n}$, with a traceless matrix $\Lambda$, induce a global $\operatorname{SL}(N)$ symmetry acting as

$$
\delta_{\Lambda} V_{m}{ }^{a}=\Lambda_{m}^{n} V_{n}{ }^{a}, \quad \delta_{\Lambda} B_{\mu}^{m}=-\Lambda_{n}^{m} B_{\mu}^{n},
$$

on the components of (4.2). Diffeomorphisms corresponding to constant rescaling of the $N$-torus, $\xi^{m}=\lambda y^{m}$, are slightly more delicate. A priori they induce an action 4.5 with diagonal matrix $\Lambda$. But as they also induce an action on the $D$-dimensional vielbein $e_{\mu}{ }^{\alpha}$, they do not constitute an off-shell symmetry in $D$ dimensions. However, combined with a proper rescaling of the $(D+N)$-dimensional vielbein 4.2 they result in an off-shell GL(1) symmetry

$$
\delta_{\lambda} \phi=\lambda N(D-2), \quad \delta_{\lambda} B_{\mu}^{m}=-\lambda(D-2+N) B_{\mu}^{m}
$$

of the $D$-dimensional theory, that leaves $e_{\mu}{ }^{\alpha}$ invariant. Comparing (4.3), (4.5), and (4.6) to the transformations (2.5), 2.24) above, we identify the scalar fields as described by an $\mathrm{GL}(N) / \mathrm{SO}(N)$ coset space $\sigma$-model with the $N$ vector fields transforming in the fundamental representation of the global symmetry $\operatorname{GL}(N)$. The resulting Lagrangian takes the form (2.4), 2.25), discussed in sections 2.1 and 2.2 above, with the matrix $\mathcal{M}_{m n} \equiv \mathrm{e}^{2(D-2+N) /(D-2) \phi} V_{m}{ }^{a} V_{n}{ }^{b} \delta_{a b}$. 
This completes the structure of pure gravity reduced on an $N$-torus. The reductions of extended supergravities typically exhibit larger global symmetry groups into which the $\mathrm{GL}(N)$ is embedded as a subgroup. The enhancement of the symmetry group is related to the presence of additional $p$-form fields in the higher-dimensional theory. The reduction ansatz for these forms is straightforward

$$
A_{M_{1} \ldots M_{p}} \longrightarrow\left(A_{\mu_{1} \ldots \mu_{p}}, A_{m_{1} \mu_{2} \ldots \mu_{p}}, A_{m_{1} m_{2} \mu_{3} \ldots \mu_{p}}, \ldots, A_{m_{1} \ldots m_{p}}\right),
$$

in terms of $D$-dimensional $p$-forms, $(p-1)$-forms, $(p-2)$-forms, etc. The transformation behavior of these fields under the $\operatorname{SL}(N)$ from (4.5) follows from their index structure in the internal indices $m_{1}, m_{2}, \ldots$, while for their scaling under the GL(1) of (4.6) one obtains (see e.g. [13])

$$
\delta_{\lambda} A_{m_{1} \cdots m_{k} \mu_{k+1} \cdots \mu_{p}}=\lambda((D-2) k+(k-p) N) A_{m_{1} \cdots m_{k} \mu_{k+1} \cdots \mu_{p}} .
$$

In particular, for $N \geq p$ the reduction 4.7 adds $\left(\begin{array}{l}N \\ p\end{array}\right)$ scalar fields $A_{m_{1} \ldots m_{p}}$ to the $D$ dimensional theory. The higher-dimensional tensor gauge transformations $\delta A_{M_{1} \ldots M_{p}}=$ $p \partial_{\left[M_{1}\right.} \Xi_{\left.M_{2} \ldots M_{p}\right]}$ which are linear in the compactified coordinates, $\Xi_{m_{2} \ldots m_{p}}=\xi_{m_{1} \ldots m_{p}} y^{m_{1}}$, induce additional global shift symmetries

$$
\delta_{\xi} A_{m_{1} \ldots m_{p}}=\xi_{m_{1} \ldots m_{p}},
$$

on these scalar fields.

A final source for scalar fields in the reduced theories are the $(D-2)$ forms that arise in the reduction (4.7) (and for $D=3$ also as the Kaluza-Klein vector fields in (4.2)). As discussed in general in section 2.2 above, in $D$-dimensions these forms can be dualized into scalar fields. It is important to note that due to their definition also these scalar fields $\varphi_{a}$ obtained by dualization possess an additional global shift symmetry

$$
\delta_{\zeta} \varphi_{a}=\zeta_{a} .
$$

Together, the symmetries directly inherited from $(D+N)$ dimensions thus form a non-semisimple group of the type $\operatorname{GL}(N) \ltimes \mathcal{N}$, with nilpotent $\mathcal{N}$ combining the shifts (4.9), 4.10). Typically, these symmetries just form an upper (Borel) half of the full semi-simple global symmetry group $\mathrm{G}$ which may be sketched as

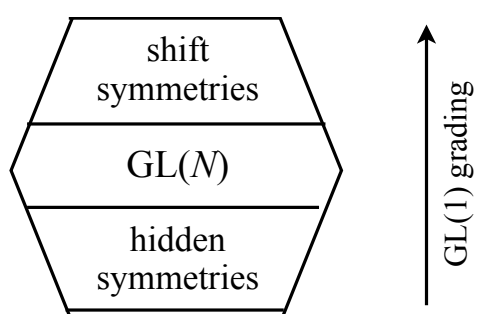

In particular, the $D$-dimensional theory typically possesses a number of $(\operatorname{dim} \mathcal{N}$, to be precise) additional symmetries - often referred to as hidden symmetries - that have 
no obvious higher-dimensional origin, and together with $\operatorname{GL}(N) \ltimes \mathcal{N}$ form the semisimple group G. The decomposition (4.11) is along the grading induced by the GL(1) scaling (4.6). The fact that the number of additional hidden symmetries is precisely enough in order to form a semi-simple global symmetry group in $D$ dimensions of course heavily hinges on the field content of the higher-dimensional supergravity theory. This is where the underlying supersymmetric structure that is preserved throughout the reduction comes to play its role. In the following we shall just make use of this matter of fact for the maximal and the half-maximal supergravities. We finally note that the decomposition 4.11 naturally selects a Borel subalgebra and thus a particular triangular gauge 2.13 for the coset space, in which the higher-dimensional origin of the $D$-dimensional scalar fields becomes most transparent. Again, we refer to [32] for a systematic discussion of the maximal supergravities in various dimensions and their eleven-dimensional origin.

One of the simplest examples of such a reduction is the Kaluza-Klein compactification of simple $D=5$ supergravity on a circle $S^{1}$. The bosonic field content of minimal $D=5$ supergravity comprises the metric and a single vector field which upon reduction 4.2, 4.7) give rise to gravity coupled to two vectors and two scalar fields. According to the discussion above, there are two global symmetries in the fourdimensional theory that are inherited from five dimensions: the GL(1) scaling (4.6) and the shift symmetry 4.9 acting on the $A_{5}$ component of the five-dimensional vector field. The full global symmetry group in four dimensions is an SL(2), which decomposes as 4.11 with each block generated by a single generator. This precisely corresponds to the example discussed at the end of section 2.1 with the two scalars parametrizing the coset space $\mathrm{SL}(2) / \mathrm{SO}(2)$ and the generators $\mathbf{h}$, e, and $\mathbf{f}$, of $\mathrm{SL}(2)$ corresponding to the scaling, the shift and the hidden symmetry, respectively.

A very different example leading to the same global symmetry group SL(2) is provided by the $S^{1}$ reduction of Einstein gravity in four dimensions. While the scaling symmetry is still (4.6), the shift symmetry is of the type 4.10 and acts on the scalar that is obtained by dualizing the three-dimensional Kaluza-Klein vector. It is in this model, that the non-linear action of a hidden symmetry in gravity (the generator $\mathbf{f}$ in this example) has first been discovered [69].

To finish this section, let us note that the action of the GL(1) scaling symmetry 4.6, 4.8 is straightforwardly extended onto those components of the higherdimensional field strength that may serve as flux parameters according to (4.1). In particular, one finds that

$$
\delta_{\lambda} \mathcal{F}_{m_{1} \cdots m_{p}}=\lambda((D-2) p+N) \mathcal{F}_{m_{1} \cdots m_{p}}
$$

for those $p$-form field strengths with all indices along the compactified directions. This will be relevant in the next sections in order to identify the proper flux parameters among the components of the embedding tensor. Similarly, we will in the following consider the theories obtained by compactification in the presence of torsion on the internal torus, i.e. by a deformation of the reduction ansatz 4.2 to $E^{a}=\tilde{E}_{m}{ }^{a}(x) \eta^{m}(y)$ 
in the internal part with the one-forms $\eta^{m}(y)$ satisfying [70, 71]

$$
d \eta^{k}=\mathcal{T}_{m n}^{k} \eta^{m} \wedge \eta^{n}
$$

with non-vanishing $\mathcal{T}_{m n}^{k}$ antisymmetric in the lower indices - often referred to as geometric flux. Analogously to 4.12, one finds for the GL(1) scaling behavior of these components

$$
\delta_{\lambda} \mathcal{T}_{m n}^{k}=\lambda(D-2+N) \mathcal{T}_{m n}^{k}
$$

\subsection{M-theory fluxes}

As a first example, we will study the reduction of eleven-dimensional supergravity [7] on a seven-torus $T^{7}$ in the presence of fluxes. This example has been analyzed in detail e.g. in [72], [73, 74, 75], and [76] The bosonic field content of the eleven-dimensional theory is the metric and an antisymmetric three-form tensor. In absence of fluxes, the reduction leads to the maximal four-dimensional ungauged supergravity [10] which has appeared on various occasions in these lectures and carries 28 electric vector fields and 70 scalars described by the coset space $\mathrm{E}_{7(7)} / \mathrm{SU}(8)$.

According to the discussion in the previous section, the first step in understanding the eleven-dimensional origin of the four-dimensional fields consists of decomposing the four-dimensional global symmetry group $\mathrm{E}_{7(7)}$ under the torus GL(7). In terms of GL(7) representations, the decomposition (4.11) takes the form

$$
\begin{aligned}
& 7^{\prime}+4 \\
& \mathrm{E}_{7(7)} \longrightarrow \frac{\frac{35_{+2}}{\mathbf{1}_{0}+\mathbf{4 8 _ { 0 }}}}{\mathbf{3 5 ^ { \prime } - 2}},
\end{aligned}
$$

with subscripts indicating the charge under GL(1) $\subset \mathrm{GL}(7)$ and the $\mathbf{1}_{0}+\mathbf{4 8}_{0}$ representing the adjoint of GL(7). The $\mathbf{3 5}_{+2}$ nilpotent symmetries in 4.15 correspond to shifts 4.9 on the 35 scalar fields descending from the eleven-dimensional three-form. The $7_{+4}^{\prime}$ shift symmetries act according to 4.10 on the scalars that are obtained by dualizing the 7 two-form fields that descend from the eleven-dimensional three-form. Their charges can be matched with (4.8) (upon choosing $\lambda=\frac{1}{3}$ ).

The 56 vector fields of the four-dimensional theory decompose according to

$$
56 \rightarrow 7_{-3}^{\prime}+21_{-1}+21_{+1}^{\prime}+7_{+3}
$$

and with the charges from (4.6), 4.8) one identifies the $\mathbf{7}_{-3}^{\prime}$ and the $\mathbf{2 1}_{-1}$ as the vector fields descending from the eleven-dimensional metric and the three-form, respectively. The other fields in (4.16) represent their magnetic duals in accordance with the discussion of section 2.3 . 
Let us now consider the possible fluxes that can be switched on in this reduction and their effect in the four-dimensional theory. The eleven-dimensional three-form tensor field can acquire a four-form flux 4.1

$$
\mathcal{F}_{n_{1} n_{2} n_{3} n_{4}}^{(4)}=c_{n_{1} n_{2} n_{3} n_{4}}
$$

with indices $n_{1}, \ldots, n_{4}$, running over the seven coordinates of the torus. As a consequence of the duality (2.29), one may alternatively consider a non-vanishing flux for its dual seven-form field strength

$$
\mathcal{F}_{n_{1} \ldots n_{7}}^{(7)}=a \epsilon_{n_{1} \ldots n_{7}} .
$$

The effective four-dimensional actions could in principle be determined by explicitly evaluating the reduction with the ansatz (4.17), (4.18). Rather than going through this quite lengthy computation, we will directly employ the underlying symmetry structure in order to identify the corresponding theories among the general gaugings presented above. From their index structure and scaling behavior 4.12), one reads off that the flux parameters of (4.17) and (4.18) transform in the $\mathbf{3 5 _ { + 5 } ^ { \prime }}$ and $\mathbf{1}_{+7}$, respectively, of GL(7). In section 3, we have established that the general deformation of the maximal four-dimensional theory is encoded in an embedding tensor $\Theta_{M}{ }^{\alpha}$ transforming in the 912 representation of $\mathrm{E}_{7(7)}$. In order to identify the particular gaugings corresponding to the fluxes 4.17) and 4.18) we simply have to identify within the $\mathbf{9 1 2}$ these particular GL(7) representations.

Breaking the $\mathbf{9 1 2}$ according to 4.15 gives the following set of representations

$$
\begin{aligned}
& 1_{+7} \\
& 35_{+5}^{\prime} \\
& \mathbf{7}_{+3}+\mathbf{1 4 0} \mathbf{0}_{+3} \\
& 21_{+1}^{\prime}+28_{+1}^{\prime}+224_{+1}^{\prime} \\
& 21_{-1}+28_{-1}+224_{-1} \\
& \mathbf{7}_{-3}^{\prime}+\mathbf{1 4 0}_{-3}^{\prime} \\
& 35_{-5} \\
& 1_{-7}
\end{aligned}
$$

in which we clearly identify the seven-form flux and the four-form flux as the upper two lines. Also the third line allows for a straightforward interpretation: the $\mathbf{7}+\mathbf{1 4 0}$ of $\mathrm{GL}(7)$ corresponds to a tensor with index structure $\mathcal{T}_{m n}^{k}$ and thus precisely to the torsion or geometric flux introduced in (4.13). The resulting four-dimensional theories can thus be obtained by evaluating the general Lagrangian sketched in section 3.3 (and given in detailed form in [42]) for the particular embedding tensor $\Theta_{M}{ }^{\alpha}$, that correspond to the upper lines of (4.19).

Gaugings that are triggered by an embedding tensor corresponding to the lower entries in 4.19) in contrast do not have a clear origin in the eleven-dimensional theory. Some of these may however find a higher-dimensional interpretation in different 
compactifications (such as the type IIB theory considered in the next section) or hint to the existence of certain non-geometric compactifications (see e.g. [77, 78, 79]).

It is important to remember that the restriction of the embedding tensor to the $\mathbf{9 1 2}$ representation in fact only represented part of the consistency constraints imposed in the four-dimensional theory. As we have discussed in section 3.1, it has to be supplemented with the quadratic constraint (3.6) in order to define a consistent gauging. In the present context this constraint translates into certain bilinear conditions on the flux parameters $a, c_{k l m n}, \mathcal{T}_{m n}^{k}$, that have to be imposed for consistency. Indeed, such conditions typically arise in the explicit study of flux compactifications. A straightforward way to obtain these bilinear conditions in our framework would be the explicit decomposition of (3.6) under GL(7). The computation may be drastically simplified by making use of a very compact way to reformulate the quadratic constraint in the four-dimensional theory. Namely, one may show that for $\Theta_{M}{ }^{\alpha}$ restricted to the $\mathbf{9 1 2}$ representation of $\mathrm{E}_{7(7)}$, the quadratic constraint (3.6) can be written in the equivalent form

$$
\Theta_{M}^{\alpha} \Theta_{N}^{\beta} \Omega^{M N}=0
$$

with the symplectic matrix $\Omega^{M N}$ of 2.34$) \cdot{ }^{12}$ This form of the constraint immediately shows that the embedding tensor considered as a matrix $\Theta_{M}{ }^{\alpha}$ has at most half-maximal rank, i.e. that the gauging involves at most 28 out of the 56 possible vector fields. More specifically, it guarantees the mutual locality of electric and magnetic charges involved in the gauging.

In order to derive possible bilinear relations between the flux parameters, it is thus useful to explicitly consider the embedding tensor $\Theta_{M}{ }^{\alpha}$ as a matrix according to the decomposition 4.15), 4.16) which yields

\begin{tabular}{|c|c|c|c|c|c|c|}
\hline$\Theta_{M}^{\alpha}$ & $7_{-4}$ & $\mathbf{3 5} \mathbf{5}_{-2}^{\prime}$ & $48_{0}$ & $\mathbf{1}_{0}$ & $\mathbf{3 5}+2$ & $\mathbf{7}_{+4}^{\prime}$ \\
\hline $\mathbf{7}_{-3}^{\prime}$ & $\mathbf{1}_{-7}$ & $35_{-5}$ & $\left(140^{\prime}+\mathbf{7}^{\prime}\right)_{-3}$ & $\mathbf{7}_{-3}^{\prime}$ & $(21+224)_{-1}$ & $\left(\mathbf{2 8 ^ { \prime }}+\mathbf{2 1 ^ { \prime }}\right)_{+1}$ \\
\hline $\mathbf{2 1}-1$ & $35-5$ & $140_{-3}^{\prime}$ & $(21+28+224)_{-1}$ & $\mathbf{2 1}-1$ & $\left(21^{\prime}+224^{\prime}\right)+1$ & $(140+7)+3$ \\
\hline $\mathbf{2} \mathbf{1}_{+1}^{\prime}$ & $\left(140^{\prime}+\mathbf{7}^{\prime}\right)_{-3}$ & $(21+224)_{-1}$ & $\left(21^{\prime}+28^{\prime}+224^{\prime}\right)_{+1}$ & $\mathbf{2} \mathbf{1}_{+1}^{\prime}$ & $140_{+3}$ & $\mathbf{3 5} \mathbf{5}_{+5}^{\prime}$ \\
\hline $7+3$ & $(\mathbf{2 8}+\mathbf{2 1})_{-1}$ & $\left(21^{\prime}+224^{\prime}\right)+1$ & $(140+7)_{+3}$ & $7_{+3}$ & $\mathbf{3 5} \mathbf{5}_{+5}^{\prime}$ & $\mathbf{1}_{+7}$ \\
\hline
\end{tabular}

with all entries built from the blocks of 4.19). In particular, coinciding representations in the bulk of the table correspond to the same flux parameters of 4.19) where they all appear with multiplicity one. It remains to evaluate the quadratic constraint 4.20) for this matrix.

To begin with, let us consider the seven-form flux represented by the $\mathbf{1}_{+7}$ which makes a single appearance in (4.21). The triangular form of this matrix shows that 4.20 is automatically satisfied, i.e. the seven-form flux defines a consistent oneparameter deformation. It is amusing to note that this particular theory has been

\footnotetext{
${ }^{12}$ This equivalence can be proven by showing that both expressions live in the same $\mathbf{1 3 3}+\mathbf{8 6 4 5}$ representation of $\mathrm{E}_{7(7)}$, see [42] for details.
} 
constructed even before the first maximal gauged supergravity of [1] was found, however in a form where the gauging is hidden in topologically massive two-forms [80].

Next, we may study gaugings induced by the four-form flux $c_{k l m n}$ which induces two entries in (4.20). Again, the triangular form of the resulting matrix (4.21) guarantees (4.20) without further constraints on $c_{k l m n}$. The first non-trivial constraint is met for gaugings induced by geometric fluxes $\mathcal{T}_{k l}^{m}$. Inspection of the associated matrix (4.21) shows that the condition 4.20 has a non-trivial component if the free indices $\alpha$ and $\beta$ take values in the $\mathbf{7}_{+4}^{\prime}$ and the $\mathbf{3 5}_{+2}$ - while the internal index $M, N$ is contracted over the $\mathbf{2} \mathbf{1}_{-1}$. The resulting constraint thus lives in the $\mathbf{7}^{\prime} \otimes \mathbf{3 5}$ by which it is entirely determined to be

$$
\mathcal{T}_{k l}^{p} \mathcal{T}_{m p}^{q}+\mathcal{T}_{l m}^{p} \mathcal{T}_{k p}^{q}+\mathcal{T}_{m k}^{p} \mathcal{T}_{l p}^{q}=0
$$

One recognizes the standard Jacobi identity, and indeed the $\mathcal{T}_{k l}^{m}$ precisely appear as structure constants (3.14) of the local gauge algebra [71, 72, 74, 76]. By similar arguments, one derives the mixed constraint

$$
\mathcal{T}_{k l}^{p} c_{m n r s} \epsilon^{q k l m n r s}-\mathcal{T}_{k l}^{q} c_{m n r s} \epsilon^{p k l m n r s}=0
$$

for gaugings that arise from simultaneous presence of four-form and geometric fluxes. Both equations (4.22) and (4.23) have non-trivial solutions. We have thus identified the relevant flux parameters within the 912 components of the general embedding tensor and derived the full set of quadratic consistency relations among them. To complete the analysis it remains to evaluate the full Lagrangian of [42] and in particular the scalar potential (3.45) for these particular embedding tensors, which we will not do here, see [81, 82] for some results.

In principle, the very same analysis can be continued for those gaugings induced by the lower lying entries of (4.19). However, the structure of the matrix (4.21) shows that the resulting quadratic constraints will be more and more involved - and thus presumably admit less and less solutions.

\subsection{IIA/IIB fluxes}

Finally, we will consider flux compactifications of the ten-dimensional type IIA/IIB theories on a six-torus $T^{6}$. These compactifications have been exhaustively studied in the literature in particular in the context of $\mathcal{N}=2$ and $\mathcal{N}=4$ supergravity, see e.g. [83, 84, 85, 86, 5] and references therein. Type IIB flux compactifications in the context of maximal supergravity that we sketch here, have been studied in [87]. We should stress that although the presence of fluxes necessarily breaks maximal supersymmetry, we may still obtain a maximally supersymmetric four-dimensional Lagrangian, in which supersymmetry is broken spontaneously in the ground state.

In order to identify the ten-dimensional origin of the four-dimensional fields, the relevant subgroups of $\mathrm{E}_{7(7)}$ are the products of the torus $\mathrm{GL}(6)$ with the global symmetry groups GL(1) and $\mathrm{SL}(2)$, respectively, of the ten-dimensional theories. The 
corresponding decompositions 4.11 take the form

$$
\begin{aligned}
& \mathbf{1}_{+3} \\
& \mathbf{6}_{+5 / 2}^{\prime} \\
& \mathbf{2 0}_{+3 / 2} \\
& 15_{+1}
\end{aligned}
$$

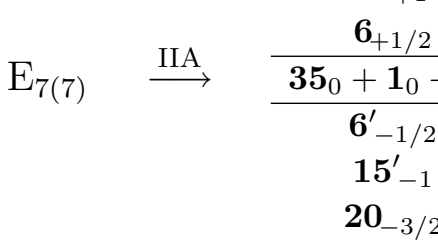

$$
\begin{aligned}
& \mathbf{6}_{-5 / 2} \\
& 1_{3}
\end{aligned}
$$

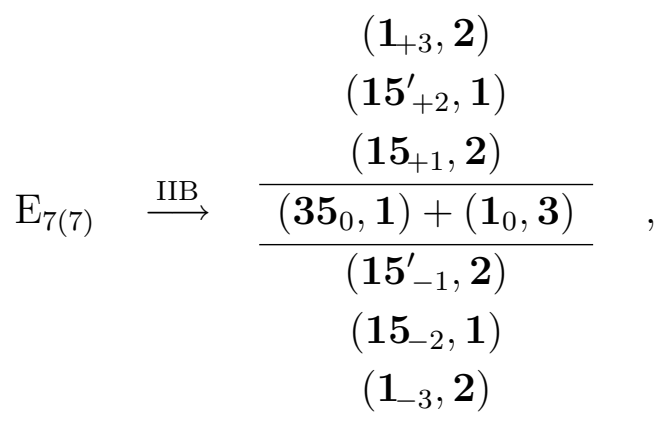

for the type IIA and IIB theory, respectively. The second number in the IIB components refers to the corresponding $\mathrm{SL}(2)$ representations. Using (4.8), it is straightforward to identify the nilpotent symmetries with positive GL(1) charge with the shift symmetries 4.9), 4.10 inherited from the higher-dimensional field content. E.g. the highest $\mathbf{1}_{+3}$ in both decompositions corresponds to the shift 4.10) acting on the scalar obtained by dualizing the two-form that descends from the ten-dimensional two-form (which is an SL(2) doublet in the IIB theory). Again these decompositions define a triangular gauge 2.13) with respect to the corresponding Borel subalgebras, in which the tendimensional origin of the fields is manifest.

In order to identify the ten-dimensional flux parameters within the $\mathbf{9 1 2}$ components of the embedding tensor we will have to decompose the latter representation under the different GL(6) subgroups of (4.24). The result is collected in figures $2 \mathrm{~A}, 2 \mathrm{~B}$, where the vertical axis corresponds to the $\mathrm{GL}(1) \subset \mathrm{GL}(6)$ grading related to rescaling of the sixtorus. Moreover, in these figures we have made the action of the $\mathrm{S}$ - and T-duality groups explicit. While the dashed diagonal lines denote orbits under the $\mathrm{SO}(6,6)$ T-duality subgroup of $\mathrm{E}_{7(7)}$, orbits under the SL(2) S-duality group correspond to horizontal lines in the IIB picture. This allows to directly read off the action of the duality groups on the various flux parameters.

As in the previous example, the components of the embedding tensor with highest values of the GL(1) grading correspond to $p$-form fluxes in ten dimensions. In the IIA picture one recognizes the six-form, the four-form, the three-form, and the twoform flux, transforming as 1, 15', 20 and 15, respectively, with their GL(1) charges given by (4.12). In the IIB decomposition, the two top rows correspond to the fiveform flux $\mathbf{6}^{\prime}$ and the three-form flux doublet $(\mathbf{2 0 , 2})$, respectively. In both diagrams, the $\mathbf{8 4}+\mathbf{6}$ appearing in the following row, denote the parameters corresponding to geometric flux $\mathcal{T}_{m n}^{k}$. As in the last section, evaluating the quadratic constraint 4.20 leads to bilinear conditions on the flux parameters, such as

$$
\epsilon^{k l m n p q} H_{k l m}^{\alpha} H_{n p q}^{\beta}=0
$$

for the three-form flux components in the IIB theory. This condition is well known [84] 


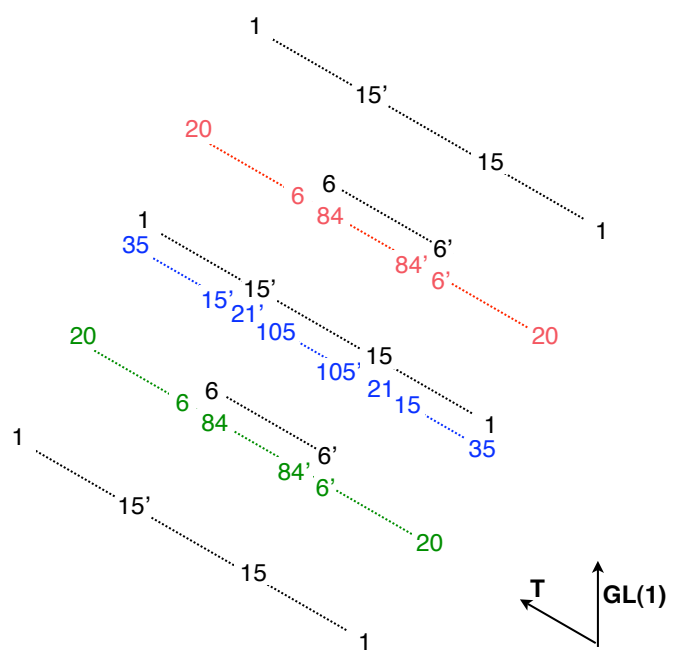

Figure 2A: The IIA flux diamond.

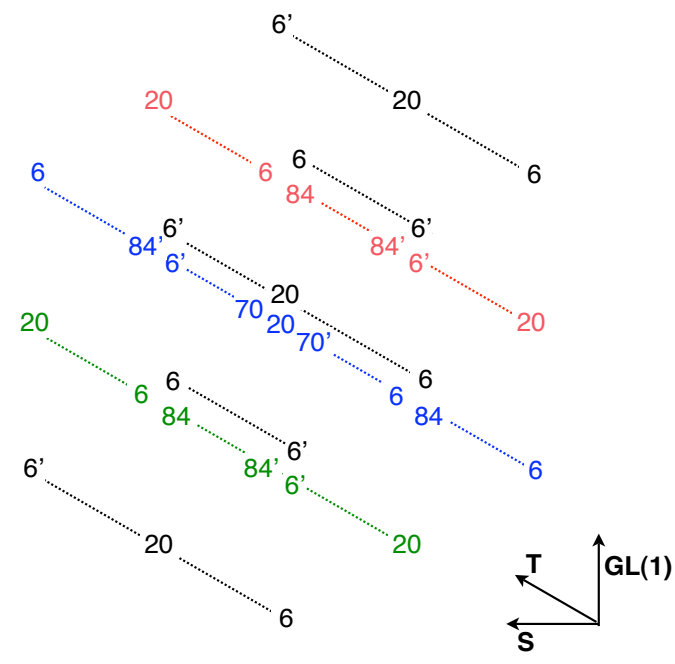

Figure 2B: The IIB flux diamond.

and usually modified by the presence of local sources which explicitly break maximal supersymmetry.

Again, the lower entries in figures 2A, 2B correspond to components of the embedding tensor whose higher-dimensional origin is less obvious. As can be seen in the figures, they may be reached by subsequent T- and S-duality transformations starting from known $p$-form and geometric flux configurations. An interesting example is the T-duality chain

$$
H_{k m n} \longrightarrow \mathcal{T}_{m n}^{k} \longrightarrow P_{k}^{m n} \longrightarrow R^{k m n}
$$

corresponding to the diagonal chain $20 \rightarrow(6+84) \rightarrow\left(6^{\prime}+84^{\prime}\right) \rightarrow 20$, which has been studied in [88]. Subsequent application of T-dualities leads from $p$-form flux $H_{k m n}$ to geometric flux $\mathcal{T}^{k}{ }_{m n}$ and beyond, to configurations parametrized by tensors $P_{k}{ }^{m n}$ and $R^{k m n}$ which have been identified as so-called non-geometric fluxes. Furthermore, figure 2B shows that the parameter $P_{k}^{m n}$ is in fact part of an S-duality doublet $\left(Q_{k}^{m n}, P_{k}^{m n}\right)$ corresponding to the horizontal pair $\left(6^{\prime}+84^{\prime}, 6^{\prime}+84^{\prime}\right)$. Indeed, this has been identified and studied in detail in [89]. While the usual approach to these non-geometric configurations is an explicit evaluation of the relevant $\mathrm{T}$ - and $\mathrm{S}$-duality transformations, we see that the covariant scheme discussed in these lectures provides a framework to construct all four-dimensional theories corresponding to the various entries in the flux diamonds of figures 2 in a closed and manifestly $E_{7(7)}$ covariant form. In particular, all bilinear conditions among the various flux parameters combine into the single equation 4.20) and the full scalar potential for generic (geometric and non-geometric) fluxes is given by the universal expression (3.45). Upon further orientifold projections, it is then possible to obtain a variety of non-maximal theories. It remains to study the properties of these theories and in particular the scalar potential for the various flux compactifications. What we have tried to illustrate here is that the covariant formulation of gauged 
supergravities provides a universal framework in which the effective theories associated with particular flux compactifications can be conveniently constructed and analyzed.

Acknowledgements: I wish to thank the organizers and participants of the RTN Winter School on Strings, Supergravity and Gauge Theories at CERN for the opportunity to present these lectures and for many comments and suggestions. It is a great pleasure to thank Eric Bergshoeff, Gianguido Dall'Agata, Bernard de Wit, Olaf Hohm, Axel Kleinschmidt, Arnaud Le Diffon, Hermann Nicolai, Nicholas Prezas, Diederik Roest, Ergin Sezgin, Mario Trigiante, and Martin Weidner, for the numerous exciting discussions and collaboration on the topics presented. This work is in part supported by the Agence Nationale de la Recherche (ANR).

\section{References}

[1] B. de Wit and H. Nicolai, $N=8$ supergravity, Nucl. Phys. B208 (1982) 323.

[2] C. M. Hull, Noncompact gaugings of $N=8$ supergravity, Phys. Lett. B142 (1984) 39; More gaugings of $N=8$ supergravity, Phys. Lett. B148 (1984) 297-300.

[3] M. Günaydin, L. J. Romans, and N. P. Warner, Compact and noncompact gauged supergravity theories in five-dimensions, Nucl. Phys. B272 (1986) 598.

[4] M. Pernici, K. Pilch, and P. van Nieuwenhuizen, Gauged maximally extended supergravity in seven-dimensions, Phys. Lett. B143 (1984) 103.

[5] M. Graña, Flux compactifications in string theory: A comprehensive review, Phys. Rept. 423 (2006) 91-158, hep-th/0509003.

[6] R. Blumenhagen, B. Kors, D. Lust and S. Stieberger, Four-dimensional string compactifications with D-Branes, orientifolds and fluxes, Phys. Rept. 445 (2007) 1 hep-th/0610327.

[7] E. Cremmer, B. Julia, and J. Scherk, Supergravity theory in 11 dimensions, Phys. Lett. B76 (1978) 409-412.

[8] J. H. Schwarz and P. C. West, Symmetries and transformations of chiral $N=2$ $D=10$ supergravity, Phys. Lett. B126 (1983) 301.

[9] P. S. Howe and P. C. West, The complete $N=2, D=10$ supergravity, Nucl. Phys. B238 (1984) 181.

[10] E. Cremmer and B. Julia, The SO(8) supergravity, Nucl. Phys. B159 (1979) 141.

[11] H. Nicolai and H. Samtleben, Maximal gauged supergravity in three dimensions, Phys. Rev. Lett. 86 (2001) 1686-1689, hep-th/0010076. 
[12] H. Nicolai and H. Samtleben, Compact and noncompact gauged maximal supergravities in three dimensions, JHEP 04 (2001) 022, hep-th/0103032.

[13] B. de Wit, H. Samtleben, and M. Trigiante, On Lagrangians and gaugings of maximal supergravities, Nucl. Phys. B655 (2003) 93-126, hep-th/0212239.

[14] B. de Wit, H. Samtleben, and M. Trigiante, The maximal $D=5$ supergravities, Nucl. Phys. B716 (2005) 215-247, hep-th/0412173.

[15] B. de Wit and H. Samtleben, Gauged maximal supergravities and hierarchies of nonabelian vector-tensor systems, Fortschr. Phys. 53 (2005) 442-449, hep-th/0501243.

[16] J. Louis and A. Micu, Type II theories compactified on Calabi-Yau threefolds in the presence of background fluxes, Nucl. Phys. B635 (2002) 395-431, hep-th/0202168.

[17] M. Graña, J. Louis, and D. Waldram, SU(3) x SU(3) compactification and mirror duals of magnetic fluxes, JHEP 04 (2007) 101, hep-th/0612237.

[18] R. D'Auria, S. Ferrara, and M. Trigiante, On the supergravity formulation of mirror symmetry in generalized Calabi-Yau manifolds, Nucl. Phys. B780 (2007) 28-39, hep-th/0701247.

[19] O. Aharony, S. S. Gubser, J. M. Maldacena, H. Ooguri, and Y. Oz, Large $N$ field theories, string theory and gravity, Phys. Rept. 323 (2000) 183-386, hep-th/9905111.

[20] L. Girardello, M. Petrini, M. Porrati, and A. Zaffaroni, Novel local CFT and exact results on perturbations of $\mathrm{N}=4$ super Yang-Mills from AdS dynamics, JHEP 12 (1998) 022, hep-th/9810126.

[21] D. Z. Freedman, S. S. Gubser, K. Pilch, and N. P. Warner, Renormalization group flows from holography supersymmetry and a $c$-theorem, Adv. Theor. Math. Phys. 3 (1999) 363-417, hep-th/9904017.

[22] M. Bianchi, D. Z. Freedman, and K. Skenderis, Holographic renormalization, Nucl. Phys. B631 (2002) 159-194, hep-th/0112119.

[23] T. Damour, M. Henneaux, and H. Nicolai, $\mathrm{E}_{10}$ and a 'small tension expansion' of M theory, Phys. Rev. Lett. 89 (2002) 221601, hep-th/0207267.

[24] P. C. West, E $E_{11}$ and M theory, Class. Quant. Grav. 18 (2001) 4443-4460, hep-th/0104081.

[25] F. Riccioni and P. West, The $\mathrm{E}_{11}$ origin of all maximal supergravities, JHEP 07 (2007) 063, arXiv:0705.0752 [hep-th].

[26] E. A. Bergshoeff, I. De Baetselier, and T. A. Nutma, $E_{11}$ and the embedding tensor, JHEP 09 (2007) 047, arXiv:0705.1304 [hep-th].

[27] P. Van Nieuwenhuizen, Supergravity, Phys. Rept. 68 (1981) 189-398. 
[28] Y. Tanii, Introduction to supergravities in diverse dimensions, hep-th/9802138.

[29] P. Fré, Gaugings and other supergravity tools of $p$-brane physics, Lectures given at Workshop on Latest Development in M-Theory, Paris, France (2001) hep-th/0102114.

[30] B. de Wit, Supergravity, in Unity from Duality: Gravity, Gauge Theory and Strings (C. Bachas, A. Bilal, F. David, M. Douglas, and N. Nekrasov, eds.), Springer, 2003. hep-th/0212245.

[31] A. Van Proeyen, Structure of supergravity theories, in Proceedings of 11th Fall Meeting on Geometry and Physics, Oviedo, Spain, Publications of the Royal Spanish Mathematical Society, 2003. hep-th/0301005.

[32] E. Cremmer, B. Julia, H. Lu, and C. N. Pope, Dualisation of dualities. I, Nucl. Phys. B523 (1998) 73-144, hep-th/9710119.

[33] A. Salam and E. Sezgin, D = 8 supergravity, Nucl. Phys. B258 (1985) 284.

[34] M. K. Gaillard and B. Zumino, Duality rotations for interacting fields, Nucl. Phys. B193 (1981) 221.

[35] B. Julia, Infinite Lie algebras in physics, in Johns Hopkins Workshop on Current Problems in Particle Theory, 1981.

[36] P. Breitenlohner and D. Maison, On the Geroch group, Ann. Poincaré 46 (1987) 215.

[37] H. Nicolai, The integrability of $N=16$ supergravity, Phys. Lett. B194 (1987) 402.

[38] H. Nicolai and H. Samtleben, Integrability and canonical structure of $d=2, N=16$ supergravity, Nucl. Phys. B533 (1998) 210-242, hep-th/9804152.

[39] D. Bernard and B. Julia, Twisted self-duality of dimensionally reduced gravity and vertex operators, Nucl. Phys. B547 (1999) 427-470, hep-th/9712254.

[40] R. Slansky, Group theory for unified model building, Phys. Rept. 79 (1981) 1-128.

[41] M. van Leeuwen, A. Cohen, and B. Lisser, LiE, a computer algebra package for Lie group computations. Computer Algebra Nederland, 1992.

[42] B. de Wit, H. Samtleben, and M. Trigiante, The maximal $D=4$ supergravities, JHEP 06 (2007) 049, arXiv:0705.2101 [hep-th].

[43] A. Le Diffon and H. Samtleben, Supergravities without an action: gauging the trombone, to appear.

[44] P. S. Howe, N. D. Lambert, and P. C. West, A new massive type IIA supergravity from compactification, Phys. Lett. B416 (1998) 303-308, hep-th/9707139.

[45] I. V. Lavrinenko, H. Lu, and C. N. Pope, Fibre bundles and generalised dimensional reductions, Class. Quant. Grav. 15 (1998) 2239-2256, hep-th/9710243. 
[46] H. Nicolai and H. Samtleben, $N=8$ matter coupled $\mathrm{AdS}_{3}$ supergravities, Phys. Lett. B514 (2001) 165-172, hep-th/0106153.

[47] J. Schön and M. Weidner, Gauged $N=4$ supergravities, JHEP 05 (2006) 034, hep-th/0602024.

[48] M. Weidner, Gauged supergravities in various spacetime dimensions, Fortsch. Phys. 55 (2007) 843-945, hep-th/0702084.

[49] E. A. Bergshoeff, J. Gomis, T. A. Nutma, and D. Roest, Kac-Moody spectrum of (half-)maximal supergravities, JHEP 02 (2008) 069, [arXiv:0711.2035 [hep-th]].

[50] B. de Wit, H. Samtleben, and M. Trigiante, Magnetic charges in local field theory, JHEP 09 (2005) 016, hep-th/0507289.

[51] J. De Rydt, T. T. Schmidt, M. Trigiante, A. Van Proeyen and M. Zagermann, Electric/magnetic duality for chiral gauge theories with anomaly cancellation, arXiv:0808.2130 [hep-th]].

[52] R. D'Auria, L. Sommovigo, and S. Vaula, $N=2$ supergravity Lagrangian coupled to tensor multiplets with electric and magnetic fluxes, JHEP 11 (2004) 028, hep-th/0409097.

[53] P. K. Townsend, K. Pilch, and P. van Nieuwenhuizen, Selfduality in odd dimensions, Phys. Lett. 136B (1984) 38.

[54] G. Dall'Agata, C. Herrmann, and M. Zagermann, General matter coupled $N=4$ gauged supergravity in five dimensions, Nucl. Phys. B612 (2001) 123-150, hep-th/0103106.

[55] L. J. Romans, Massive $N=2 a$ supergravity in ten-dimensions, Phys. Lett. B169 (1986) 374.

[56] B. de Wit, H. Nicolai, and H. Samtleben, Gauged supergravities, tensor hierarchies, and M-theory, JHEP 02 (2008) 044, arXiv:0801.1294 [hep-th]].

[57] F. Riccioni and P. West, $E_{11}$-extended spacetime and gauged supergravities, JHEP 02 (2008) 039, arXiv:0712.1795 [hep-th].

[58] H. Samtleben and M. Weidner, The maximal $D=7$ supergravities, Nucl. Phys. B 725 (2005) 383, hep-th/0506237.

[59] M. de Roo, D. B. Westra, and S. Panda, Gauging CSO groups in $N=4$ supergravity, JHEP 09 (2006) 011, hep-th/0606282.

[60] J.-P. Derendinger, P. M. Petropoulos, and N. Prezas, Axionic symmetry gaugings in $N=4$ supergravities and their higher-dimensional origin, Nucl. Phys. B785 (2007) 115-134, arXiv:0705.0008 [hep-th].

[61] H. Samtleben and M. Weidner, Gauging hidden symmetries in two dimensions, JHEP 08 (2007) 076, arXiv:0705.2606 [hep-th]]. 
[62] M. de Vroome and B. de Wit, Lagrangians with electric and magnetic charges of $N=2$ supersymmetric gauge theories, JHEP 08 (2007) 064, arXiv:0707.2717 [hep-th]].

[63] E. Bergshoeff, H. Samtleben, and E. Sezgin, The gaugings of maximal $D=6$ supergravity, JHEP 03 (2008) 068, [arXiv:0712.4277 [hep-th]].

[64] E. Bergshoeff, T. de Wit, U. Gran, R. Linares, and D. Roest, (Non-)Abelian gauged supergravities in nine dimensions, JHEP 10 (2002) 061, hep-th/0209205.

[65] E. Bergshoeff, U. Gran, R. Linares, M. Nielsen, T. Ortin, and D. Roest, The Bianchi classification of maximal $D=8$ gauged supergravities, Class. Quant. Grav. 20 (2003) 3997-4014, hep-th/0306179.

[66] J. A. Strathdee, Extended Poincaré supersymmetry, Int. J. Mod. Phys. A2 (1987) 273.

[67] B. de Wit, I. Herger, and H. Samtleben, Gauged locally supersymmetric $D=3$ nonlinear sigma models, Nucl. Phys. B671 (2003) 175-216, hep-th/0307006.

[68] E. A. Bergshoeff, M. de Roo, S. F. Kerstan, and F. Riccioni, IIB supergravity revisited, JHEP 08 (2005) 098, hep-th/0506013.

[69] J. Ehlers, Konstruktion und Charakterisierungen von Lösungen der Einsteinschen Gravitationsgleichungen. PhD thesis, University of Hamburg, 1957.

[70] J. Scherk and J. H. Schwarz, How to get masses from extra dimensions, Nucl. Phys. B153 (1979) 61-88.

[71] N. Kaloper and R. C. Myers, The $\mathrm{O}(\mathrm{dd})$ story of massive supergravity, JHEP 05 (1999) 010, hep-th/9901045.

[72] G. Dall'Agata and S. Ferrara, Gauged supergravity algebras from twisted tori compactifications with fluxes, Nucl. Phys. B717 (2005) 223-245, hep-th/0502066.

[73] L. Andrianopoli, M. A. Lledó, and M. Trigiante, The Scherk-Schwarz mechanism as a flux compactification with internal torsion, JHEP 05 (2005) 051, hep-th/0502083.

[74] R. D'Auria, S. Ferrara, and M. Trigiante, $E_{7(7)}$ symmetry and dual gauge algebra of M-theory on a twisted seven-torus, Nucl. Phys. B732 (2006) 389-400, hep-th/0504108.

[75] R. D'Auria, S. Ferrara, and M. Trigiante, Supersymmetric completion of M-theory $4 D$-gauge algebra from twisted tori and fluxes, JHEP 01 (2006) 081, hep-th/0511158.

[76] C. M. Hull and R. A. Reid-Edwards, Flux compactifications of M-theory on twisted tori, JHEP 10 (2006) 086, hep-th/0603094.

[77] C. M. Hull, A geometry for non-geometric string backgrounds, JHEP 10 (2005) 065, hep-th/0406102. 
[78] C. M. Hull and R. A. Reid-Edwards, Gauge symmetry, T-duality and doubled geometry, arXiv:0711.4818 [hep-th].

[79] G. Dall'Agata, N. Prezas, H. Samtleben, and M. Trigiante, Gauged supergravities from twisted doubled tori and non-geometric string backgrounds, Nucl. Phys. B799 (2008) 80-109, arXiv:0712.1026 [hep-th].

[80] A. Aurilia, H. Nicolai, and P. K. Townsend, Hidden constants: The theta parameter of QCD and the cosmological constant of $N=8$ supergravity, Nucl. Phys. B176 (1980) 509.

[81] R. D'Auria, S. Ferrara, and M. Trigiante, Curvatures and potential of M-theory in $D=4$ with fluxes and twist, JHEP 09 (2005) 035, hep-th/0507225.

[82] G. Dall'Agata and N. Prezas, Scherk-Schwarz reduction of M-theory on G2-manifolds with fluxes, JHEP 10 (2005) 103, hep-th/0509052.

[83] S. B. Giddings, S. Kachru, and J. Polchinski, Hierarchies from fluxes in string compactifications, Phys. Rev. D66 (2002) 106006, hep-th/0105097.

[84] S. Kachru, M. B. Schulz, and S. Trivedi, Moduli stabilization from fluxes in a simple IIB orientifold, JHEP 10 (2003) 007, hep-th/0201028.

[85] R. D'Auria, S. Ferrara, F. Gargiulo, M. Trigiante, and S. Vaula, $N=4$ supergravity Lagrangian for type IIB on $T^{6} / Z_{2}$ in presence of fluxes and D3-branes, JHEP 06 (2003) 045, hep-th/0303049.

[86] C. Angelantonj, S. Ferrara and M. Trigiante, Unusual gauged supergravities from type IIA and type IIB orientifolds, Phys. Lett. B 582 (2004) 263 hep-th/0310136.

[87] B. de Wit, H. Samtleben, and M. Trigiante, Maximal supergravity from IIB flux compactifications, Phys. Lett. B583 (2004) 338-346, hep-th/0311224.

[88] J. Shelton, W. Taylor, and B. Wecht, Nongeometric flux compactifications, JHEP 10 (2005) 085, hep-th/0508133.

[89] G. Aldazabal, P. G. Camara, A. Font, and L. E. Ibanez, More dual fluxes and moduli fixing, JHEP 05 (2006) 070, hep-th/0602089. 